\title{
Surface subgroups and handlebody attachment
}

\author{
VIVIEN R EASSON
}

\begin{abstract}
The main theorem of this paper generalizes recent results in Dehn surgery to the case of handlebody attachment. We consider attaching handlebodies and solid tori to the boundary of an irreducible, boundary-irreducible, atoroidal and acylindrical 3-manifold. We show that for a large class of homeomorphisms attaching these handlebodies, the fundamental group of the resulting manifold contains the fundamental group of a closed surface of genus at least two.
\end{abstract}

57N10; 57M50, 57N35, 20H10

\section{Introduction}

In this paper, we discuss a particular generalization of the technique of Dehn surgery for hyperbolic 3-manifolds, which we call handlebody attachment. We consider a simple 3-manifold $M$ (not $B^{3}$ ) with non-empty boundary, where simple means compact, irreducible, $\partial$-irreducible, atoroidal and acylindrical.

As a consequence of the proof of Thurston's geometrization of Haken manifolds, we can give $M$ a complete hyperbolic structure such that the non-cuspidal part of $M$ is compact and every boundary component of $M$ which has genus at least two inherits a totally geodesic metric from the hyperbolic structure on $M$. When we use the term totally geodesic boundary throughout this paper, this is what we mean. Thus the only non-compact ends of $M$ are cusps.

We consider attaching a collection of handlebodies and solid tori $\mathcal{H}$ to $\partial M$. Denote the resulting manifold by $M \cup_{\phi} \mathcal{H}$, where $\phi=\left\{\phi_{\ell}\right\}$ is a collection of homeomorphisms, and each $\phi_{\ell}$ is a map from a boundary component $(\partial M)_{\ell}$ of $\partial M$ to the boundary of a handlebody or solid torus $H_{\ell}$ in $\mathcal{H}$.

A surface subgroup of $M$ is a subgroup $\Gamma \leq \pi_{1}(M)$ which is isomorphic to the fundamental group of a closed surface of genus at least two. In [8], Cooper-Long-Reid showed that $M$ always has a non-peripheral surface subgroup.

In the Dehn surgery case, $\mathcal{H}$ is a collection of solid tori. Recent papers of CooperLong [7] and Li [16] showed independently that all but finitely many Dehn surgeries 
on a one-cusped hyperbolic manifold give rise to a manifold with a surface subgroup. Bart [2] gives a result when there are many cusps. We generalize these results to the case of handlebody attachments.

For a handlebody $H \in \mathcal{H}$, we consider attachments involving pseudo-Anosov homeomorphisms $h: \partial H \rightarrow \partial H$ whose stable lamination is of full type with respect to some pants decomposition of $H$ along meridians, see Definition 2.7. This condition is generically true, and is equivalent (see [1]) to the condition that $h$ has stable lamination which lies in the Masur domain of the handlebody.

Theorem 1 (Theorems 6.1 and 7.1) Suppose $M \neq B^{3}$ is a simple 3-manifold with $m+m^{\prime} \geq 1$ boundary components, precisely $m^{\prime}$ of which are tori. Take a collection $\mathcal{H}=\left\{H_{1}, \ldots, H_{m+m^{\prime}}\right\}$ of handlebodies and solid tori whose genera match those of $\partial M$. Let $M \cup_{\phi} \mathcal{H}$ denote the closed 3-manifold obtained by gluing each boundary component $(\partial M)_{\ell}$ to $\partial H_{\ell}$ by a homeomorphism $\phi_{\ell}$.

Suppose moreover that $h_{\ell}: \partial H_{\ell} \rightarrow \partial H_{\ell}$ is a homeomorphism which is either a pseudoAnosov homeomorphism whose stable lamination is of full type, or an Anosov homeomorphism, according to whether $H_{\ell}$ has genus at least two or is a solid torus respectively.

Given homeomorphisms $\phi_{\ell}^{\prime}:(\partial M)_{\ell} \rightarrow \partial H_{\ell}$, there exist integers $\left(N_{\ell}\right)_{\min }$ such that if $\phi_{\ell}=h_{\ell}^{N_{\ell}} \circ \phi_{\ell}^{\prime}$ with $N_{\ell} \geq\left(N_{\ell}\right)_{\min }$ for all $\ell$, the group $\pi_{1}\left(M \cup_{\phi} \mathcal{H}\right)$ contains a surface subgroup.

There are three main ingredients required in our proof of Theorem 1. Firstly, a closed $\pi_{1}$-injective surface $\Sigma$ with a finite cover which lifts to an embedded incompressible surface in a finite cover of $M$. Secondly, suitable classes of gluing homeomorphisms. Finally, a geometrical analysis of the ways in which $\Sigma$ might fail to remain $\pi_{1}$-injective under handlebody attachment.

We first prove Theorem 1 assuming $M$ has no toral boundary components: every component of $\partial M$ is a surface of genus at least two. This proof is given in Sections 2 to 6, and generalized to the case where $M$ also has some toral boundary components in Section 7. The precise method of proof when the chosen hyperbolic metric on $M$ has cusps depends on whether $\Sigma$ has accidental parabolics, but is broadly similar to the non-cusped case.

In both cases, we prove Theorem 1 as a corollary of the following theorem.

Theorem 2 (Theorems 6.2 and 7.2) Let $M$ be a hyperbolic 3-manifold with $m$ totally geodesic boundary components and $m^{\prime}$ cusps, $m+m^{\prime} \geq 1$. Let $\Sigma$ be a 
connected, orientable, closed, immersed surface in $M$ with a finite cover which lifts to a non-peripheral embedded incompressible surface in a finite regular cover $\pi: \widetilde{M} \rightarrow M$ of degree $d$.

As in Theorem 1, let $M \cup_{\phi} \mathcal{H}$ denote the closed 3-manifold obtained by attaching handlebodies to $\partial M$ via homeomorphisms $\phi_{\ell}:(\partial M)_{\ell} \rightarrow \partial H_{\ell}$ and consider maps $h_{\ell}$ satisfying the condition given in that theorem.

Given homeomorphisms $\phi_{\ell}^{\prime}:(\partial M)_{\ell} \rightarrow \partial H_{\ell}$, there exist integers $\left(N_{\ell}\right)_{\min }$ such that if $\phi_{\ell}=h_{\ell}^{N_{\ell}} \circ \phi_{\ell}^{\prime}$ with $N_{\ell} \geq\left(N_{\ell}\right)_{\min }$ for all $\ell$, the surface $\Sigma$ remains $\pi_{1}$-injective in the resulting manifold $M \cup_{\phi} \mathcal{H}$.

The existence of such a surface $\Sigma$ is given by Cooper-Long-Reid [8]. However our proof works for any virtually embedded $\pi_{1}$-injective surface satisfying the conditions above, not just those provided by their construction.

The second ingredient is making a good choice of attaching maps. The crucial property possessed by each gluing homeomorphism described above is that its dynamics are governed by that of the pseudo-Anosov map. In Section 2 we explain the condition for a lamination to be of full type, in terms of the way it intersects pants decompositions of the handlebody.

When we attach handlebodies by maps as above, we force each member of a certain finite collection of curves to have high geometric intersection number with every curve bounding a disc in a handlebody $H$ under the attachment map. Furthermore, we can use the geometry of these intersection sets to prove a key lemma, Proposition 4.1.

Now we use our final ingredient. Suppose that our surface $\Sigma$ fails to inject at the level of the fundamental group into $M \cup_{\phi} \mathcal{H}$. Then there exists an essential loop $\mathcal{L}$ in $\Sigma$, and an immersed disc $D$ in $M \cup_{\phi} \mathcal{H}$ which spans $\mathcal{L}$. The intersection of $D$ with $M$ is a planar surface, and we may consider an immersed least-area representative $Q$ of this planar surface. By the Gauss-Bonnet theorem, the area of $Q$ is bounded above by a linear function of its Euler characteristic, and all but one component of $\partial Q$ bound a disc in $\mathcal{H}$ under the attaching maps.

Our proof relies on showing that the intersections as above contribute area to a surface $R$. This surface is obtained as a cover of $Q$ of degree at most $d$, where $d$ is the degree of a finite cover $\pi: \widetilde{M} \rightarrow M$ in which $\Sigma$ becomes embedded. For a sufficiently high power of the pseudo-Anosov map, the intersections contribute too much area, and we obtain a contradiction with the Gauss-Bonnet theorem.

We develop this intuition in Sections 2 to 6 in order to prove Theorem 2 and hence Theorem 1 in the case when every boundary component of $M$ is of genus at least 
two. In Section 7 we show that the presence of cusps does not materially affect our argument, so that Theorems 1 and 2 remain true in the general case.

Note that attaching a solid torus by a map as in the theorems ensures that the corresponding Dehn filling slope lies outside the union of a finite number of infinite strips. In fact a similar argument to ours shows that our results hold in the latter case as well. The important condition turns out to be that the Dehn filling slope should have high intersection number with a finite number of exceptional slopes. These slopes depend only on the surface $\Sigma$ and on the way in which its convex hull intersects the boundary of $M$.

I would like to thank my DPhil supervisor Marc Lackenby for a great many helpful conversations. My thanks also to Juan Souto for helping me to place my original condition on the pseudo-Anosov maps in the context of the literature, to Saul Schleimer for comments on terminology, and to the referee for several helpful suggestions.

\section{Defining the projective wavelike set}

Let $H$ be a handlebody of genus $g$, and write $B(\partial H)_{\text {emb }}$ for the collection of nontrivial embedded 1-manifolds on $\partial H$, each component of which bounds a disc in $H$, considered up to isotopy. Similarly, let $B(\partial H)$ denote the collection of non-trivial regular homotopy classes of closed curves on $\partial H$, not necessarily embedded, each component of which bounds a disc in $H$.

We will consider the first set as a subset of $M L(\partial H)$, measured laminations on $\partial H$, by giving each curve component unit weight. The second set may be thought of in terms of Bonahon's theory of geodesic currents (e.g. [5]), but this is not necessary for our discussion.

Definition 2.1 Suppose $\mathcal{P}$ is a pants decomposition of the surface $\partial H$ such that every boundary component of a pair of pants $P \in \mathcal{P}$ lies in $B(\partial H)_{\mathrm{emb}}$. Then we say that $\mathcal{P}$ is a handlebody pants decomposition and that every $P \in \mathcal{P}$ is an $H$-pants.

We will find it useful to distinguish two different kinds of essential subarcs in an $H$-pants $P$, and relate this to the way that a lamination $\lambda$ intersects $P$.

Definition 2.2 Suppose that $P$ is a pair of pants, and that $\xi$ is an essential subarc properly embedded in $P$. If both of the endpoints of $\xi$ lie on the same boundary component of $P$, we say that $\xi$ is a wave in $P$. Otherwise, $\xi$ connects two different boundary components of $P$, and we say that $\xi$ is a seam in $P$. 
Similarly, an essential immersed subarc $\xi$ with $\xi \cap \partial P=\partial \xi$ is said to be an immersed wave or an immersed seam according to whether its endpoints lie on the same or different components of $\partial P$.

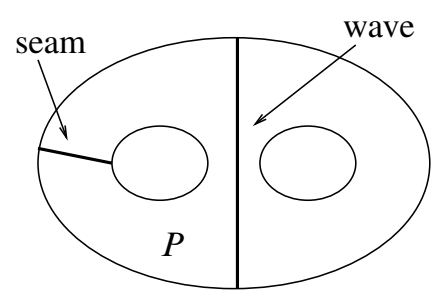

Figure 1: Waves and seams in a pair of pants

Definition 2.3 Consider a measured lamination $\lambda$ and an $H$-pants $P$. Label the boundary components of $P$ by $\partial P_{1}, \partial P_{2}, \partial P_{3}$. Let $a, b, c$ denote respectively $a=$ $\left|\lambda \cap \partial P_{1}\right|, b=\left|\lambda \cap \partial P_{2}\right|$ and $c=\left|\lambda \cap \partial P_{3}\right|$, where $|x \cap y|$ denotes the geometric intersection number of measured laminations $x$ and $y$.

Then $\lambda$ satisfies all triangle inequalities on $P$ if $a+b \geq c, b+c \geq a$ and $c+a \geq b$. We say that $\lambda$ satisfies all strict triangle inequalities if every such inequality is strict.

On the other hand, if at least one of these strict inequalities does not hold (e.g. $a+b \leq c$ ), we say that $\lambda$ satisfies some triangle subequality. Thus it is possible (take $a+b=c$, $b+c \geq a, c+a \geq b$ ) that $\lambda$ satisfies both all triangle inequalities and some triangle subequality.

We can show that curves in $B(\partial H)$ usually have an immersed wave with respect to some component of a handlebody pants decomposition.

Lemma 2.4 Consider a closed, connected, possibly non-embedded curve $\alpha$ which lies in the boundary of a handlebody $H$ and is homotopically trivial in $H$. Let $\mathcal{P}$ be a handlebody pants decomposition of $\partial H$. Then either $\alpha$ is homotopic into some component $P \in \mathcal{P}$ or $\alpha$ has an immersed wave with respect to some pants $P \in \mathcal{P}$.

Proof Suppose some component of $\alpha$ is not homotopic into any component $P \in \mathcal{P}$, and write $f: D \rightarrow H$ for an immersed disc in $H$ that it bounds. Then by ambient isotopy of $f(D)$ we may assume it intersects the discs in $\mathcal{P}$ in a minimal non-empty collection of arcs whose preimages are properly and disjointly embedded in $D$. An outermost such preimage $\operatorname{arc} c$ separates off a subdisc $f: D^{\prime} \rightarrow H$ with boundary 

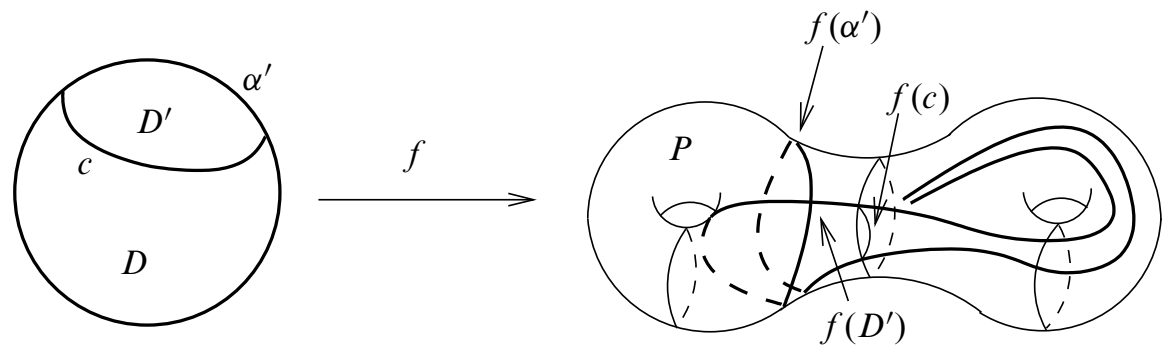

Figure 2: A wave in a curve bounding a disc

$c \cup \alpha^{\prime}$ whose image lies in the union of some $H$-pants $P$ and the discs in $H$ bounded by its boundary components.

Since the endpoints of $f(c)$ lie in the same boundary component of $P$, so do those of $f\left(\alpha^{\prime}\right)$. By minimality, $f\left(\alpha^{\prime}\right)$ is an essential arc which is proper and immersed in $P$, so it forms an immersed wave in $P$.

For $\alpha$ embedded, this wave will be embedded, and we can say more.

Corollary 2.5 Suppose that $\alpha \in B(\partial H)_{\mathrm{emb}}$. Then the following holds for any handlebody pants decomposition $\mathcal{P}$, whether or not there is a wave. There exist an $H$-pants $P \in \mathcal{P}$, and two boundary components $\partial P_{1}$ and $\partial P_{2}$ of $P$, such that $\alpha$ has no subarcs in $P$ which are seams connecting $\partial P_{1}$ and $\partial P_{2}$.

Proof If $\alpha$ has a wave, it cannot have some seam which would cross that wave; and if $\alpha$ lies inside $P$, it has a component parallel to a boundary component of some $P$ and so cannot have any seam running from that component.

Moreover, suppose $\alpha \in B(\partial H)_{\mathrm{emb}}$ has $k>0$ waves in $P$ joining $\partial P_{3}$ to itself. These also separate $\partial P_{1}$ from $\partial P_{2}$, so we deduce that the corresponding triple of intersection numbers is of the form $(a, b, c)=(a, b, a+b+2 k)$. If $\alpha$ has no waves in $P$ but some component of $\alpha$ is parallel to $\partial P_{1}$, we have $(a, b, c)=(0, b, b)$.

In particular, given any handlebody pants decomposition $\mathcal{P}$, and a curve $\alpha$ in the set $B(\partial H)_{\mathrm{emb}}$, there exists an $H$-pants $P \in \mathcal{P}$ such that $\alpha$ satisfies a triangle subequality on $P$. We are interested in all curves and laminations satisfying this conclusion, which motivates the following definition.

Definition 2.6 Define the wavelike set $\mathcal{W}(H) \subset M L(\partial H)$ to be $\mathcal{W}(H)=\{\lambda: \forall \mathcal{P}, \exists P \in \mathcal{P}$ s.t. $\lambda$ satisfies some triangle subequality on $P\}$ 
where $\mathcal{P}$ ranges over all handlebody pants decompositions of $H$.

Since a measured lamination $\lambda$ lies in $\mathcal{W}(H)$ if and only if any positive scalar multiple of $\lambda$ does, we can also define the projective wavelike set $P \mathcal{W}(H)$ consisting of all projective measured laminations which have a representative in $\mathcal{W}(H)$. This is a subset of $\operatorname{PML}(\partial H)$, projective measured lamination space. We may therefore make the following definition.

Definition 2.7 Let $\lambda$ be a measured lamination in $M L(\partial H)$. If it does not lie in $\mathcal{W}(H)$ then we say it is of full type. Similarly, a projective measured lamination is of full type if it does not lie in $P \mathcal{W}(H)$.

Suppose $h: \partial H \rightarrow \partial H$ is a pseudo-Anosov homeomorphism whose stable and unstable laminations $\lambda^{S}$ do not lie in the projective wavelike set $P \mathcal{W}(H)$. Then we say that $h$ is of full type.

We showed above that $B(\partial H)_{\mathrm{emb}} \subset \mathcal{W}(H)$. Abrams-Schleimer proved that $P \mathcal{W}(H)$ is equal to the closure of $P B(\partial H)_{\mathrm{emb}}$ in the subspace of $P M L(\partial H)$ consisting of those laminations which occur as the fixed points of pseudo-Anosov homeomorphisms (see [1], 11.5). They also demonstrated that $h$ has stable lamination of full type if and only if $h$ has stable lamination contained in the Masur domain of $H$ : the set of laminations having non-zero geometric intersection with every lamination in the closure of $P B(\partial H)_{\mathrm{emb}}$.

By work of Kerckhoff [13] which extends results of Masur [17], the closure of $P B(\partial H)_{\mathrm{emb}}$ and hence $P \mathcal{W}(H)$ has measure zero in $P M L(\partial H)$. Thus a generic pseudo-Anosov map is of full type. The terminology full type comes from Kobayashi [14].

We will consider attaching maps of the form $\phi=h^{N} \circ \phi^{\prime}$ which are formed as the composition of a power of a pseudo-Anosov homeomorphism $h: \partial H \rightarrow \partial H$ whose stable lamination is of full type and a homeomorphism $\phi^{\prime}:(\partial M)_{\ell} \rightarrow \partial H$. Our results hold for all $\phi$ with $N$ larger than some constant depending on $h$ and $\phi^{\prime}$.

We use throughout the conventions for stable and unstable laminations of a pseudoAnosov map found in [6]. In Section 4, we show how to put these definitions together with that of the set of distinguished curves defined below.

\section{The set of distinguished curves $X_{i}$}

We start by defining what it means for a 3-manifold to be simple. Then, given such a manifold $M$ (with non-empty boundary) and a suitable $\pi_{1}$-injective surface $\Sigma$ 
immersed in it, we define sets $X_{i}$ of distinguished curves which will play a crucial role in our argument.

Definition 3.1 An orientable 3-manifold $M$ is said to be simple if it is compact, irreducible, $\partial$-irreducible, atoroidal and acylindrical.

Take a simple 3-manifold $M$ with $m$ boundary components of genus at least two and $m^{\prime}$ toral boundary components, $m+m^{\prime} \geq 1$. As a consequence of the proof of Thurston's geometrization theorem for Haken 3-manifolds, we can give $M$ (minus its toral boundary) a complete hyperbolic structure $g$ such that the non-cuspidal part of $M$ is compact and every boundary component of genus at least two inherits a totally geodesic hyperbolic metric from it.

For $M$ as above, we will consider an immersed surface $\eta: \Sigma \rightarrow M$ which is is a closed, connected, orientable $\pi_{1}$-injective surface of genus at least two and which has a finite cover $\widetilde{\Sigma}$ lifting to a non-peripheral embedded incompressible surface in a degree $d$ cover $\pi: \widetilde{M} \rightarrow M$.

We can always assume that this cover is regular, by lifting if necessary to a further finite cover. The next result follows from various facts originally due to Thurston, as described below.

Lemma 3.2 Let $\eta: \Sigma \uparrow \rightarrow M$ be a surface in a simple 3-manifold $M$ as above. Then $\Sigma$ is geometrically finite.

Proof Suppose firstly that $M$ has at least one boundary component of genus at least two. Then, giving $M$ a hyperbolic metric $g$ as above, the limit set of the Kleinian group $\pi_{1}(M)$ in the hyperbolic sphere at infinity $S_{\infty}^{2}=\partial \overline{\mathbb{R}^{3}}$ does not fill up all of the sphere. Matsuzaki-Taniguchi [18] call this a Kleinian group of the second kind. Theorem 3.11 of [18] states that every finitely generated subgroup of such a group is geometrically finite. This fact of Thurston's can also be found in [20]. Since $\Sigma$ is a closed surface, $\pi_{1}(\Sigma)$ is finitely generated and hence geometrically finite.

On the other hand, suppose that $M$ only has toral boundary components, so that $(M, g)$ is a complete hyperbolic 3-manifold with finite volume. In this case, it follows from work of Thurston [21] and Bonahon [4] that either $\Sigma$ is geometrically finite or it is a virtual fibre of $M$ : see for example the discussion in Menasco-Reid [19]. But $\Sigma$ is closed so $M$ cannot virtually fibre over it. Therefore $\Sigma$ is geometrically finite.

Take a virtually embedded $\pi_{1}$-injective surface $\eta: \Sigma \uparrow \rightarrow M$ as above. Write $\widetilde{\Sigma}_{1}, \ldots, \widetilde{\Sigma}_{s}$ for the images of the connected surface $\widetilde{\Sigma}$ which is a cover of $\Sigma$ lifting to $\widetilde{M}$. These 
are embedded incompressible surfaces which intersect each other in $\widetilde{M}$. We shall assume these conditions and this terminology throughout this paper. Until Section 7, we also assume $m^{\prime}=0$, so $M$ has no cusps.

Let $\Psi_{i}$ denote the characteristic submanifold of $\widetilde{M}_{i}^{*}=\widetilde{M}-\operatorname{int}\left(N\left(\widetilde{\Sigma}_{i}\right)\right)$, where $\widetilde{M}_{i}^{*}$ is the manifold obtained from $\widetilde{M}$ by removing a small open neighbourhood of the embedded surface $\widetilde{\Sigma}_{i}$. Its vertical boundary $\partial_{v} \Psi_{i}$ is a collection of disjoint essential annuli properly embedded in $\left(\widetilde{M}_{i}^{*}, \partial \widetilde{M} \cup \widetilde{\Sigma}_{i}\right)$.

Moreover, since $M$ is hyperbolic, $\Psi_{i}$ consists of $I$-bundles over subsurfaces of the boundary and Seifert-fibred solid tori. This follows since $M$ being atoroidal implies that no other Seifert-fibred spaces occur as components of $\Psi_{i}$. Again, we note that the submanifolds $\Psi_{1}, \ldots, \Psi_{s}$ intersect each other.

Consider a restricted subset of the connected components of each $\Psi_{i}$. This subset consists of those $I$-bundles whose vertical boundary is a collection of annuli with one boundary component in each of $\partial \widetilde{M}$ and $\widetilde{\Sigma}_{i}$. Write $\Psi\left(\partial \widetilde{M}, \widetilde{\Sigma}_{i}\right)$ for the collection of all such $I$-bundles in $\widetilde{M}$.

The characteristic submanifold is a purely topological object, but we will impose some geometrical restrictions on the subset $\Psi\left(\partial \widetilde{M}, \widetilde{\Sigma}_{i}\right)$. We will assume that the boundary components of $\Psi\left(\partial \widetilde{M}, \widetilde{\Sigma}_{i}\right) \cap \partial \widetilde{M}$ are geodesic curves in $\partial \widetilde{M}$. This may make some previously disjoint annuli in $\partial_{v} \Psi\left(\partial \widetilde{M}, \widetilde{\Sigma}_{i}\right)$ intersect along a closed geodesic in $\partial \widetilde{M}$, as shown in Figure 3. Note that there are finitely many of these curves since each of them is the geodesic straightening of a boundary curve of the original $\Psi\left(\partial \widetilde{M}, \widetilde{\Sigma}_{i}\right) \cap \partial \widetilde{M}$. We now give a precise definition.

Definition 3.3 Suppose $x$ is a closed simple geodesic on a totally geodesic boundary component of $\partial \widetilde{M}$ with the following property. There exist curves $\sigma^{+}$and $\sigma^{-}$on $\widetilde{\Sigma}_{i}$ which are freely homotopic to $x$ in $\widetilde{M}_{i}^{*}$ but not freely homotopic to each other in $\widetilde{\Sigma}_{i}$. Then we say that $x$ is a cut curve for $\Psi\left(\partial \widetilde{M}, \widetilde{\Sigma}_{i}\right)$.

We are interested in the intersection of $\Psi\left(\partial \widetilde{M}, \widetilde{\Sigma}_{i}\right)$ with the boundary $\partial \widetilde{M}$. For our argument it is convenient to express this intersection set in terms of the convex hull $\mathcal{C}\left(\widetilde{\Sigma}_{i}\right)$ of $\widetilde{\Sigma}_{i}$. We will use the same notation $\mathcal{C}(-)$ throughout for convex hulls in $\mathbb{H}^{3}$ and their images when we project to a quotient manifold.

We will also occasionally find it useful to consider a slightly larger submanifold containing the convex hull which has smooth boundary. We may do this by extending the convex hull in a neighbourhood of small width which will be determined precisely later. To avoid excessive notation, we also let $\mathcal{C}(-)$ denote the resulting extended convex hull with smooth boundary. 
vertical boundary annuli in $\Psi\left(\partial \widetilde{M}, \widetilde{\Sigma}_{i}\right)$

(before making curves on $\partial \widetilde{M}$ geodesic)

$\left[\times S^{1}\right]$

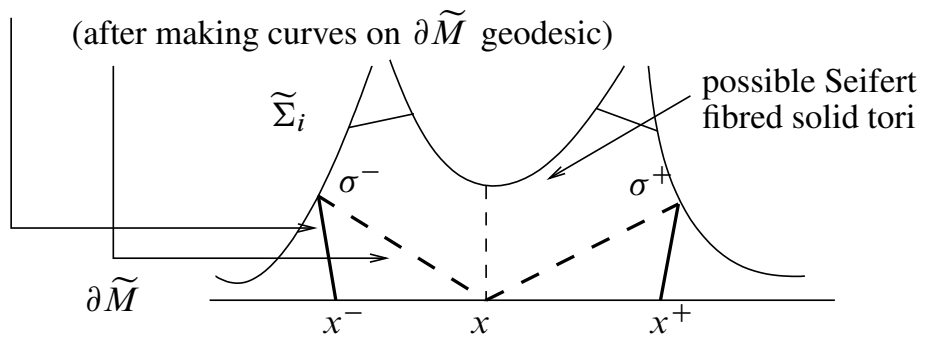

Figure 3: The curve $x$ is a cut curve in this picture $\times S^{1}$

Proposition 3.4 For $\widetilde{M}, \mathcal{C}\left(\widetilde{\Sigma}_{i}\right)$ and $\Psi\left(\partial \widetilde{M}, \widetilde{\Sigma}_{i}\right)$ as defined above,

$$
\Psi\left(\partial \widetilde{M}, \widetilde{\Sigma}_{i}\right) \cap \partial \widetilde{M}=\mathcal{C}\left(\widetilde{\Sigma}_{i}\right) \cap \partial \widetilde{M} .
$$

Proof For each boundary component $(\partial \widetilde{M})_{\ell}$ of $\widetilde{M}$, choose a basepoint $*$ on $(\partial \widetilde{M})_{\ell}$ for all fundamental groups considered. Starting from the right-hand-side of the above equation, we have

$$
\begin{aligned}
\mathcal{C}\left(\widetilde{\Sigma}_{i}\right) \cap \partial \widetilde{M} & =\bigcup_{\ell}\left(\mathcal{C}\left(\widetilde{\Sigma}_{i}\right) \cap(\partial \widetilde{M})_{\ell}\right) \\
& =\bigcup_{\ell}\left(\mathcal{C}\left(\Lambda\left(\pi_{1}\left(\widetilde{\Sigma}_{i}\right)\right)\right) \cap \mathcal{C}\left(\Lambda\left(\pi_{1}\left((\partial \widetilde{M})_{\ell}\right)\right)\right)\right),
\end{aligned}
$$

where $\Lambda(\Gamma)$ denotes the limit set in $\partial \mathbb{\boxplus}^{3}$ of a Kleinian group $\Gamma$.

The limit set of the totally geodesic surface $(\partial \widetilde{M})_{\ell}$ is a round circle. Thus the convex hull of $(\partial \widetilde{M})_{\ell}$ is a geodesic hyperplane in $\mathbb{H}^{3}$. Since the convex hull of $\widetilde{\Sigma}_{i}$ lies to one side of this hyperplane, we deduce that

$$
\mathcal{C}\left(\widetilde{\Sigma}_{i}\right) \cap(\partial \widetilde{M})_{\ell}=\mathcal{C}\left(\Lambda\left(\pi_{1}\left(\widetilde{\Sigma}_{i}\right)\right) \cap \Lambda\left(\pi_{1}\left((\partial \widetilde{M})_{\ell}\right)\right)\right) .
$$

By a standard theorem on limit sets (see for example Theorem 3.14 of [18]), the intersection of the limit sets of two geometrically finite subgroups is the limit set of their intersection, plus perhaps a set $P$ of parabolic fixed points. By our current assumption that $M$ has no cusps, $P=\varnothing$. Therefore

$$
\mathcal{C}\left(\widetilde{\Sigma}_{i}\right) \cap(\partial \widetilde{M})_{\ell}=\mathcal{C}\left(\Lambda\left(\pi_{1}\left(\widetilde{\Sigma}_{i}\right) \cap \pi_{1}\left((\partial \widetilde{M})_{\ell}\right)\right)\right) .
$$


Consider a loop $x$ on $(\partial \widetilde{M})_{\ell}$ based at $*$ which lies in $\pi_{1}\left(\widetilde{\Sigma}_{i}\right) \cap \pi_{1}\left((\partial \widetilde{M})_{\ell}\right)$. Choose a path $p$ from the basepoint $*$ on $(\partial \widetilde{M})_{\ell}$ to a point on $\widetilde{\Sigma}_{i}$. Then take a loop $\sigma$ on $\widetilde{\Sigma}_{i}$ running through this point such that $p \sigma p^{-1}$ is homotopic to $x$ relative to $*$. Use this homotopy to define an annulus $f: A \leftrightarrow \widetilde{M}$ with boundary $\sigma \cup x$, obtained by identifying two sides of the square with sides $x, p, \sigma$ and $p^{-1}$.

Put $f: A \leftrightarrow \widetilde{M}$ into general position with respect to the embedded surface $\widetilde{\Sigma}_{i}$, and assume that the annulus $f(A)$ is transverse to $\widetilde{\Sigma}_{i}$ in a neighbourhood of $f(\partial A)$. It follows that the preimage of $f(A) \cap \widetilde{\Sigma}_{i}$ consists of simple closed curves in $A$. We can remove any inessential simple closed curves $\alpha$ from $A$ since $\widetilde{\Sigma}_{i}$ is incompressible: $f(\alpha)$ will bound discs in $f(A)$ and therefore in $\widetilde{\Sigma}_{i}$; these discs cobound a ball across which $f(A)$ may be homotoped to remove the intersection.

Therefore we may assume that the preimage of $f(A) \cap \widetilde{\Sigma}_{i}$ in $A$ consists of core curves in $A$. Take the core curve $\alpha$ nearest the boundary component of $A$ mapping to $x$ in $(\partial \widetilde{M})_{\ell}$. Let $f: A_{x} \rightarrow \widetilde{M}$ denote the subannulus of $f: A \leftrightarrow \widetilde{M}$ lying between $f(\alpha)$ and $f(x)$. The interior of this annulus $f\left(A_{x}\right)$ is disjoint from $\widetilde{\Sigma}_{i}$, and so it lies in $\widetilde{M}_{i}^{*}$.

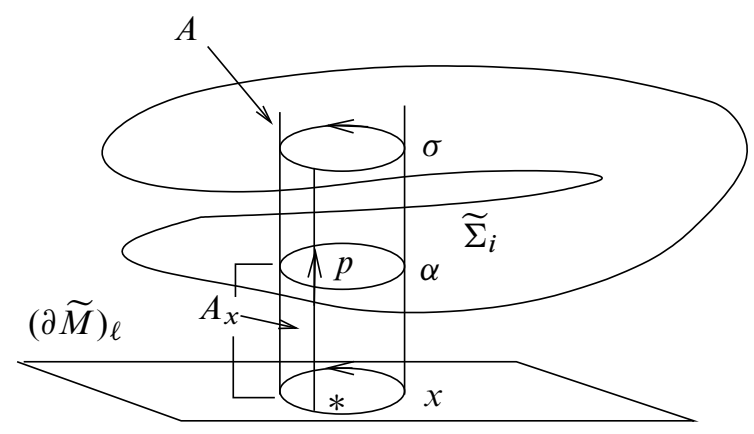

Figure 4: Homotopy annulus from $\sigma$ to $x$

Any such annulus can be homotoped into the characteristic submanifold $\Psi_{i}$ of $\widetilde{M}_{i}^{*}$ by Johannson's Enclosing Theorem [11]. By construction, it can be homotoped into the subset $\Psi\left(\partial \widetilde{M}, \widetilde{\Sigma}_{i}\right) \subset \Psi_{i}$. Thus the curve $x$ on $(\partial \widetilde{M})_{\ell}$ can be isotoped into $\Psi\left(\partial \widetilde{M}, \widetilde{\Sigma}_{i}\right) \cap(\partial \widetilde{M})_{\ell}$. Indeed, since we assumed that the boundary components of $\Psi\left(\partial \widetilde{M}, \widetilde{\Sigma}_{i}\right) \cap \partial \widetilde{M}$ were geodesic, the geodesic straightening of $x$ already lies inside the intersection $\Psi\left(\partial \widetilde{M}, \widetilde{\Sigma}_{i}\right) \cap(\partial \widetilde{M})_{\ell}$. We deduce that $\mathcal{C}\left(\widetilde{\Sigma}_{i}\right) \cap(\partial \widetilde{M})_{\ell}$ lies inside $\Psi\left(\partial \widetilde{M}, \widetilde{\Sigma}_{i}\right) \cap(\partial \widetilde{M})_{\ell}$.

Conversely, consider any loop $x$ in $\Psi\left(\partial \widetilde{M}, \widetilde{\Sigma}_{i}\right) \cap(\partial \widetilde{M})_{\ell}$ based at $*$. By definition of $\Psi\left(\partial \widetilde{M}, \widetilde{\Sigma}_{i}\right)$, it is homotopic relative to $*$ to a loop of the form $p \sigma p^{-1}$, where $p$ is 
some path from $*$ to $\widetilde{\Sigma}_{i}$ and $\sigma$ is a loop in $\widetilde{\Sigma}_{i}$. Thus $x \in \pi_{1}\left(\widetilde{\Sigma}_{i}\right) \cap \pi_{1}\left((\partial \widetilde{M})_{\ell}\right)$, and the geodesic representative of $x$ lies in

$$
\mathcal{C}\left(\widetilde{\Sigma}_{i}\right) \cap(\partial \widetilde{M})_{\ell}=\mathcal{C}\left(\Lambda\left(\pi_{1}\left(\widetilde{\Sigma}_{i}\right) \cap \pi_{1}\left((\partial \widetilde{M})_{\ell}\right)\right)\right) .
$$

Since the boundary curves of both $\Psi\left(\partial \widetilde{M}, \widetilde{\Sigma}_{i}\right) \cap(\partial \widetilde{M})_{\ell}$ and $\mathcal{C}\left(\widetilde{\Sigma}_{i}\right) \cap(\partial \widetilde{M})_{\ell}$ are geodesic, $x$ also lies inside $\mathcal{C}\left(\widetilde{\Sigma}_{i}\right) \cap(\partial \widetilde{M})_{\ell}$. This gives the opposite inclusion. By repeating both directions of this argument for every component of $\partial \widetilde{M}$, we obtain the required equality $\Psi\left(\partial \widetilde{M}, \widetilde{\Sigma}_{i}\right) \cap \partial \widetilde{M}=\mathcal{C}\left(\widetilde{\Sigma}_{i}\right) \cap \partial \widetilde{M}$.

The curves which occur either as cut curves defined in Definition 3.3 or as boundary curves of the set described in Proposition 3.4 will play a special role in our argument. Sometimes we consider some additional curves as well.

Definition 3.5 We define a boundary curve for $\widetilde{\Sigma}_{i}$ to be an essential simple closed geodesic $x$ in $\partial \widetilde{M}$ which is a component of $\partial\left(\Psi\left(\partial \widetilde{M}, \widetilde{\Sigma}_{i}\right) \cap \partial \widetilde{M}\right)$. We say that an essential simple closed geodesic in $\partial \widetilde{M}$ is a characteristic curve for $\widetilde{\Sigma}_{i}$ if it is a boundary curve for $\widetilde{\Sigma}_{i}$ or a cut curve for $\widetilde{\Sigma}_{i}$.

Let $X_{i}^{\prime}$ denote the set of characteristic curves for $\widetilde{\Sigma}_{i}$. Then $X_{i}^{\prime}$ is a possibly empty finite collection of disjoint essential simple closed geodesics on $\partial \widetilde{M}$.

Definition 3.6 Suppose that $X_{i}^{\prime} \cap(\partial \widetilde{M})_{\ell}$ were empty for some boundary component $(\partial \widetilde{M})_{\ell}$ of $\widetilde{M}$. Then we choose an essential simple closed geodesic $x_{\ell} \subset(\partial \widetilde{M})_{\ell}$ and replace $X_{i}^{\prime}$ by $X_{i}^{\prime} \cup\left\{x_{\ell}\right\}$. We call $x_{\ell}$ an additional curve.

Extend each $X_{i}^{\prime}$ to a set $X_{i}$ which is the union of $X_{i}^{\prime}$ and an additional curve $x_{\ell}$ for every boundary component requiring one. Then $X_{i} \cap(\partial \widetilde{M})_{\ell}$ is non-empty for all $i$ and $\ell$. We call $X_{i}$ the set of distinguished curves for $\Psi\left(\partial \widetilde{M}, \widetilde{\Sigma}_{i}\right)$. It consists of the characteristic curves for $\widetilde{\Sigma}_{i}$ and the additional curves chosen as above. If $x$ is a curve in $X_{i}$, we say it is a distinguished curve.

We will also require the following definition.

Definition 3.7 Let $\alpha$ be an immersed arc on $(\partial \widetilde{M})_{\ell}$. We say that $\alpha$ is a quintersecting arc if it intersects every distinguished curve $x \in \bigcup_{i} X_{i}$ on $(\partial \widetilde{M})_{\ell}$ at least five times. 


\section{Ensuring large intersection with $X_{i}$}

In this section we use the definitions above and show that certain curves whose images bound discs in an attached handlebody have large geometric intersection number with each distinguished curve in every set $X_{i}$.

Proposition 4.1 Let $M \neq B^{3}$ be a simple 3-manifold containing a closed, connected, orientable, immersed surface $\eta: \Sigma \rightarrow M$ with a finite cover which lifts to a nonperipheral embedded incompressible surface in a degree $d$ cover $\pi: \widetilde{M} \rightarrow M$. Let $H$ be a handlebody. Suppose that $h: \partial H \rightarrow \partial H$ is a pseudo-Anosov map whose stable lamination is of full type and that $\phi^{\prime}:(\partial M)_{\ell} \rightarrow \partial H$ is a homeomorphism from some boundary component $(\partial M)_{\ell}$ of $M$ to $\partial H$.

Then, for all $n \in \mathbb{N}$, there exists $N_{\min }\left(h, \phi^{\prime}\right) \in \mathbb{N}$ depending on the maps $\eta, \pi, h$ and $\phi^{\prime}$ and on the integers $n$ and $d$ such that every attaching homeomorphism $\phi=$ $h^{N} \circ \phi^{\prime}:(\partial M)_{\ell} \rightarrow \partial H$ with $N \geq N_{\min }\left(h, \phi^{\prime}\right)$ has the following property.

[*] Any connected essential (not necessarily embedded) curve $\rho \subset(\partial \widetilde{M})_{\ell}$ such that $\phi \circ \pi(\rho)$ bounds a disc in $H$ can be subdivided into at least $n$ subarcs, each of which is a quintersecting arc.

Proof Take the component of $\partial \widetilde{M}$ containing $\rho$ and denote it by $(\partial \widetilde{M})_{\ell}$. Thus $(\partial \widetilde{M})_{\ell}$ projects to $(\partial M)_{\ell}$. The homeomorphism $\phi^{\prime}:(\partial M)_{\ell} \rightarrow \partial H$ allows us to define a cover $\pi_{\phi^{\prime}}$ of $\partial H$ by

$$
\pi_{\phi^{\prime}}:=\phi^{\prime} \circ \pi: \partial \widetilde{M}_{\ell} \rightarrow \partial H
$$

We write $\partial \widetilde{H}=\partial \widetilde{M}_{\ell}$. Note that in general $\pi_{\phi^{\prime}}$ does not extend to a cover of the handlebody $H$. We then want to be able to lift a homeomorphism $h: \partial H \rightarrow \partial H$ to a homeomorphism $\widetilde{h}: \widetilde{\partial H} \rightarrow \widetilde{\partial H}$. The necessary and sufficient condition for this to be possible is that the map $h_{*}$ induced by $h$ at the level of the fundamental group should satisfy $h_{*} \Gamma=\Gamma$, where $\Gamma \leq \pi_{1}(\partial H)$ is the subgroup

$$
\Gamma=\left(\pi_{\phi^{\prime}}\right)_{*}\left(\pi_{1}(\widetilde{\partial H})\right) .
$$

This subgroup is of finite index equal to $\bar{d} \leq d$, where $d$ is the degree of the cover $\pi: \widetilde{M} \rightarrow M$. The group $\pi_{1}(\partial H)$ is finitely generated, hence it has finitely many subgroups of index $\bar{d}$, and the automorphism $h_{*}$ must permute them. Such a permutation has order $v$ dividing $t$ ! where

$$
t=\max _{\bar{d} \leq d}\left\{\text { number of subgroups of } \pi_{1}(\partial H) \text { of index } \bar{d}\right\}<\infty
$$


Thus for any $h$, the induced map $\left(h^{t !}\right)_{*}=\left(h_{*}\right)^{t !}$ preserves $\Gamma$. This implies that $h^{t !}$ lifts to a homeomorphism

$$
\widetilde{h^{t} !}: \widetilde{\partial H} \rightarrow \widetilde{\partial H} \text {. }
$$

Consider the map $\phi=h^{N} \circ \phi^{\prime}$, and take $n \in \mathbb{N}$. We may write $N$ as $N^{\prime} t !+r$, where $0 \leq r<t$ !. Write $\phi$ as

$$
\phi=\left(h^{t !}\right)^{N^{\prime}} \circ\left(h^{r} \circ \phi^{\prime}\right),
$$

noting that none of $N^{\prime}, t$ or $r$ depends on $\phi^{\prime}$.

Suppose that $\psi:(\partial M)_{\ell} \rightarrow \partial H$ ranges over the homeomorphisms in the set

$$
\Phi_{t !}=\left\{\phi^{\prime}, h \phi^{\prime}, h^{2} \phi, \ldots, h^{t !-1} \phi^{\prime}\right\} .
$$

If for every $\psi \in \Phi_{t !}$ we can obtain an integer $N_{\min }\left(h^{t !}, \psi\right)$ which satisfies the requirements of the proposition, then we can take

$$
N_{\min }\left(h, \phi^{\prime}\right)=t ! \times\left(\max _{\psi \in \Phi_{t !}} N_{\min }\left(h^{t !}, \psi\right)+1\right) .
$$

Indeed, if we take $N \geq N_{\min }\left(h, \phi^{\prime}\right)$ with $N_{\min }\left(h, \phi^{\prime}\right)$ defined as above, then for

$$
h^{N} \circ \phi^{\prime}=\left(h^{t !}\right)^{N^{\prime}} \circ\left(h^{r} \circ \phi^{\prime}\right) \text {, }
$$

we have

$$
N^{\prime} \geq \frac{N}{t !}-1 \geq N_{\min }\left(h^{t !}, \psi\right)
$$

with $\psi=h^{r} \circ \phi^{\prime}$, so that the conclusion holds.

Thus from now on we will assume that the map $h$ lifts to a pseudo-Anosov homeomorphism $\widetilde{h}: \widetilde{\partial H} \rightarrow \partial \widetilde{\partial H}$. The stable lamination $\widetilde{\lambda^{S}} S$ of $\widetilde{h}$ is $\pi_{\phi^{\prime}}^{-1}\left(\lambda^{S}\right)$.

We define $\widetilde{\phi}: \partial \widetilde{M}_{\ell} \rightarrow \widetilde{\partial H}$ to be $\widetilde{h}^{N}$ using $\widetilde{\partial H}=\partial \widetilde{M}_{\ell}$. Then

$$
\pi_{\phi^{\prime}} \circ \widetilde{\phi}=\pi_{\phi^{\prime}} \circ \widetilde{h}^{N}=h^{N} \circ \pi_{\phi^{\prime}}=h^{N} \circ \phi^{\prime} \circ \pi=\phi \circ \pi
$$

so that gluing commutes with the projection map.

By our choice of map $h, \lambda^{S} \in P \mathcal{W}(H)^{c}$. Thus the lamination $\lambda^{S}$ satisfies all strict triangle inequalities on each pants component $P$ of some handlebody pants decomposition $\mathcal{P}=\mathcal{P}\left(\lambda^{S}\right)$. As $\lambda^{S}$ has no self-intersections and never spirals into a closed leaf, the strict triangle inequalities imply that $\lambda^{S}$ has no waves and has all possible seams. Moreover, the union $\lambda^{S} \cup \mathcal{P}$ splits $\partial H$ up into simply-connected regions, either square or hexagonal.

In the cover $\widetilde{\partial H}$, these regions must lift to simply-connected regions. Figure 5 shows an example of a possible lift of some $P \in \mathcal{P}$. 


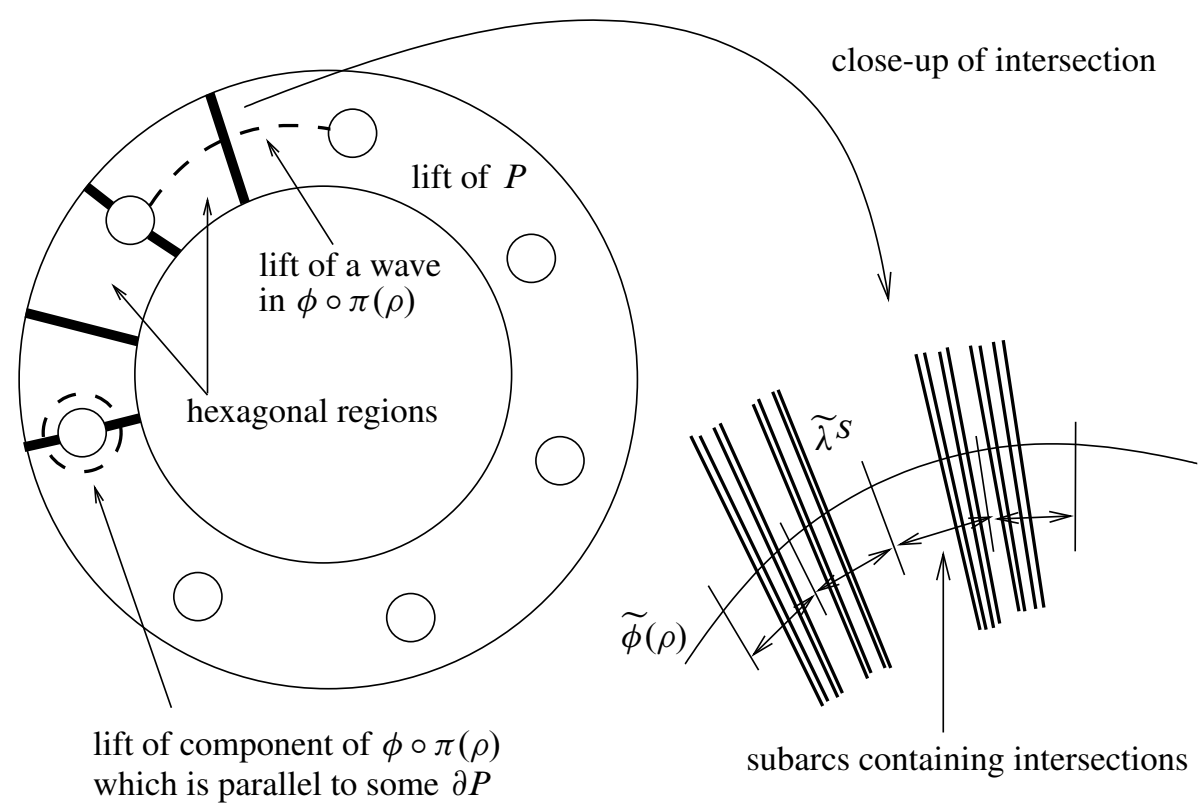

Figure 5: Every lift of a wave intersects $\widetilde{\lambda}^{S}$ many times

We now carry out the technical part of the proof. Take a curve $\rho$ in $\partial \widetilde{M}$ (connected but not necessarily simple) such that $\phi \circ \pi(\rho)=\pi_{\phi^{\prime}} \circ \widetilde{\phi}(\rho)$ bounds a disc in $H$. This disc is proper and essential in $(H, \partial H)$ but need not be embedded. By Lemma 2.4, either $\phi \circ \pi(\rho)$ is freely homotopic into $P \in \mathcal{P}$ and hence separates two boundary components of the $H$-pants $P$, or it has an immersed wave with respect to $\mathcal{P}$. In either case, $\phi \circ \pi(\rho)$ intersects $\lambda^{S}$.

This intersection persists when we lift to $\widetilde{\partial H}: \widetilde{\phi}(\rho)$ must intersect $\widetilde{\lambda}^{S}$. Indeed, the lift of the immersed wave (or boundary-parallel curve) starts in one of the square or hexagonal regions and must leave by one of the edges lying in $\widetilde{\lambda}^{S}$. Furthermore, since the lamination $\widetilde{\lambda}^{S}$ has local geometry modelled on a Cantor set $\times \mathbb{R}$, the lift of the wave intersects $\widetilde{\lambda}^{S}$ uncountably many times near this point.

Apply the map $\widetilde{\phi}=\widetilde{h}^{N}$ to a simple closed curve $x$ in $(\partial \widetilde{M})_{\ell}$. Denoting the geodesic straightening of a curve's image by $(-)^{*}$, we have

$$
(\widetilde{\phi}(x))^{*}=\left(\widetilde{h}^{N}(x)\right)^{*} \subset \widetilde{\partial H}
$$

As $N \rightarrow \infty,\left(\widetilde{h}^{N}(x)\right)^{*} \rightarrow \widetilde{\lambda}^{S}$ in the Gromov-Hausdorff sense (e.g. [12], §11.15). 
Write $W(x)$ for the smallest integer (depending on $\eta, \pi, h$ and $\phi^{\prime}$ ) such that whenever $N \geq W(x)$, the following property holds. This integer will exist by the convergence above.

Property 4.2 For every lift $\widetilde{\xi}$ of a seam $\xi$ in an $H$-pants $P$ of $\mathcal{P}$ such that $\widetilde{\lambda}^{S}$ has a subarc homotopy equivalent to $\widetilde{\xi}$ keeping its endpoints on $\partial P$, the curve $\left(\widetilde{h}^{N}(x)\right)^{*}$ has a subarc homotopy equivalent to $\widetilde{\xi}$ keeping its endpoints on $\partial P$.

Note that there may not be a uniform bound $W$ such that, for all simple closed curves $x, W(x) \leq W$. Consider however the set of distinguished curves $X_{i}$ in $\partial \widetilde{M}$ from Definition 3.6. Since there are finitely many curves in each $X_{i}$, and finitely many lifts $\widetilde{\Sigma}_{i}$ from which we obtain a set of distinguished curves $X_{i}$, there are finitely many curves in all of these sets put together.

Write $X_{(\partial \widetilde{M})_{\ell}}$ for $\cup_{i} X_{i} \cap(\partial \widetilde{M})_{\ell}$. Since $X_{(\partial \widetilde{M})_{\ell}}$ is finite, we can define $W_{X, \ell}$ to be the maximum of the integers $W(x)$ such that $x \in X_{(\partial \widetilde{M})_{\ell}}$. Then, for $N \geq W_{X, \ell}$ and a geodesic $x \in X_{(\partial \widetilde{M})_{\ell}}$, the curves

$$
\widetilde{\phi}(\rho))^{*} \equiv\left(\widetilde{h}^{N}(\rho)\right)^{*} \quad \text { and } \quad\left(\widetilde{h}^{N}(x)\right)^{*}
$$

have non-zero geometric intersection number. Thus $\rho$ and $x$ intersect, proving a weak form of the proposition.

More generally, take a point $p$ in the intersection of $\widetilde{\lambda}^{S}$ with $\widetilde{\phi}(\rho)=\widetilde{h}^{N}(\rho)$. As $N$ tends to infinity, the intersection of $(\widetilde{\phi}(x))^{*}=\left(\widetilde{h}^{N}(x)\right)^{*}$ with $\widetilde{h}^{N}(\rho)$ converges to $\widetilde{\lambda}^{S} \cap \widetilde{h}^{N}(\rho)$ around $p$ in the sense of Gromov-Hausdorff. In particular, the intersections tend to the Cantor set limit.

To complete the proof, take $n \in \mathbb{N}$. Any curve $\widetilde{\phi}(\rho)$ as above has at least $n$ subarcs each of which intersects $\widetilde{\lambda}^{S}$ at least five times, possibly all on the same lift of a wave. This implies that for each distinguished curve $x$ there exists $N_{\min }(x)$ such that $\widetilde{\phi}(\rho)$ has at least $n$ subarcs each of which intersects $\phi(x)$ at least five times.

Since there are finitely many distinguished curves and their images all converge to $\widetilde{\lambda} S$, we deduce that there exists $N_{\min }$ such that $\widetilde{\phi}(\rho)$ has $n$ subarcs each of which intersects all distinguished curves at least five times. For example, pick $5 n$ consecutive intersections between $\widetilde{\phi}(\rho)$ and $\widetilde{\lambda}^{S}$, a number $\epsilon>0$ small enough so that the distance between any two such intersections is greater than $2 \epsilon$, and take $N_{\min }$ large enough so that for every distinguished curve $x$, the geodesic $(\phi(x))^{*}$ has an intersection with $\widetilde{\phi}(\rho)$ within an $\epsilon$-neighbourhood of each intersection with $\widetilde{\lambda}^{S}$. Thus the same subarcs may be chosen so that they work for all distinguished curves. 
Moreover, the integer $N_{\min }$ can be taken to be independent of $\rho$ since there are only finitely many types of square or hexagonal regions to consider. We conclude the proof by noting that this property is preserved under $\widetilde{\phi}^{-1}$. Thus there exists $N_{\min }$ so that every $\rho$ can be subdivided into at least $n$ subarcs, each of which is quintersecting. $\square$

\section{Gathering area outside $\mathcal{C}\left(\Sigma_{i}\right)$}

In Sections 3 and 4 we obtained two useful results. Proposition 3.4 expresses the intersection of the convex hull of a surface $\widetilde{\Sigma}_{i}$ with $\partial \widetilde{M}$ as the intersection of a certain collection of $I$-bundles $\Psi\left(\partial \widetilde{M}, \widetilde{\Sigma}_{i}\right)$ with $\partial \widetilde{M}$. Proposition 4.1 gives conditions guaranteeing that certain curves in $\partial \widetilde{M}$ have many subarcs, each intersecting every curve in the set $X_{i}$ of distinguished curves for $\Psi\left(\partial \widetilde{M}, \widetilde{\Sigma}_{i}\right)$.

In this section and Section 6 we combine these results to show that all but a small number of these subarcs containing enough of these intersections will contribute a fixed quantum of area to a lift of a planar surface lying in $M$. This surface will be of the following kind.

Let $f: Q \leftrightarrow \rightarrow M$ be a least-area planar surface immersed in $M$, with $k \geq 2$ geodesic boundary components $f\left(q_{1}\right), \ldots, f\left(q_{k}\right)$ lying on $\partial M$ and a geodesic boundary component $f\left(q_{0}\right)$ lying in $\mathcal{C}(\Sigma)$ and homotopic to a curve in $\Sigma$. This surface $Q$ inherits a negatively curved metric from $M$ (see Theorem 5.5, §V.A, [9]) of curvature at most -1 . Assume also that $Q$ is homotopically incompressible and homotopically $\partial$-incompressible.

Basmajian [3] showed that the totally geodesic boundary $\partial M$ of a hyperbolic manifold such as $M$ has a collar of width some fixed constant $U$ depending only on $\left\{\chi\left((\partial M)_{\ell}\right)\right\}$. This leads us to the following definitions of a $U$-collar and of minimal $U$-thin $\operatorname{arcs}$ in a planar surface immersed in $M$.

Definition 5.1 For $M, \Sigma$ and $Q$ as above, consider a curve $q_{j}(j \neq 0)$. The $U$-collar of $q_{j}$ consists of all points $\zeta \in Q$ such that the distance $d\left(\zeta, q_{j}\right)$ is at most $U$ in the path metric on $Q$ obtained from the metric on $M$.

By Basmajian's result these collars are disjoint for $q_{j}(j \neq 0)$. For if not, we can find an embedded essential arc in $Q$ running between curves $q_{j_{1}}$ and $q_{j_{2}}$ which lies in the $U$-collar of $\partial M$ and so can be homotoped into $\partial M$. This contradicts homotopic $\partial$-incompressibility of $Q$. However, the curve $f\left(q_{0}\right)$ may approach some $f\left(q_{j}\right)$ at a distance less than $U$, and so we define $\delta$-thin $\operatorname{arcs}$ for $Q$. 
Definition 5.2 Define a minimal $U-t h i n$ arc in $f: Q \rightarrow M$ to be a geodesic arc $\xi$ properly embedded in $Q$ with endpoints on $q_{0}$ and some $q_{j}(j \neq 0)$, such that (i) the length of $\xi$ is at most $U$ in the path metric on $Q$; (ii) $\xi$ is of minimal length among embedded arcs homotopy equivalent to it keeping their endpoints on the boundary.
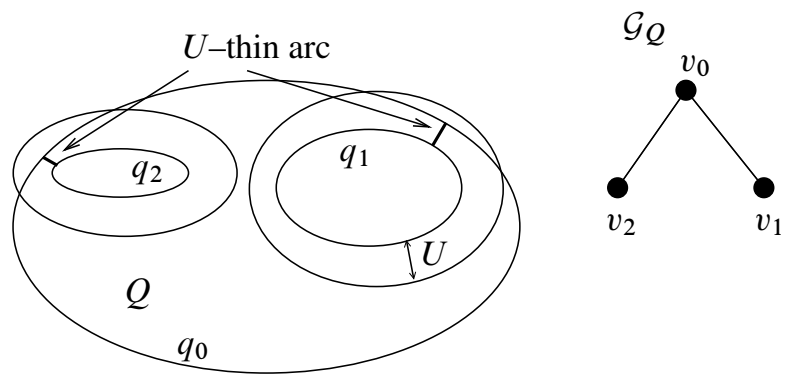

Figure 6: Definition of minimal $U$-thin arcs and example of a graph

Note that these arcs are disjoint. For any constant $0<\delta \leq U$, we may similarly define minimal ffi-thin arcs for $f: Q \rightarrow M$. Moreover, if $R$ is a cover of $Q$ which lifts to some finite cover $\widetilde{M} \rightarrow M$, the set of minimal $\delta$-thin $\operatorname{arcs}$ of $R$ consists of all arcs in $R$ which project to a minimal $\delta$-thin $\operatorname{arc}$ of $Q$.

Lemma 5.3 Let $\tilde{f}: R \leftrightarrow \widetilde{M}$ be a (minimal) surface immersed in $\widetilde{M}$ such that its projection $Q=\pi(R)$ is a least-area planar surface in $M$ as described above, with $k+1 \geq 3$ boundary components. Suppose that $d$ is the degree of the cover $\pi: \widetilde{M} \rightarrow M$. Then, for any $0<\delta \leq U$, there are at most $2(k-1) d$ minimal $\delta$-thin $\operatorname{arcs}$ in $R$.

Proof By construction it is enough to show that $Q$ has at most $2(k-1)$ minimal $\delta$-thin arcs, where $k \geq 2$. Consider therefore the graph $\mathcal{G}_{Q}$ obtained from $Q$ by taking a vertex for every boundary component of $Q$ and an edge for every minimal $\delta$-thin arc. Then $\mathcal{G}_{Q}$ is planar, and we claim it has at most $2(k-1)$ edges.

We prove this by induction on $k$. If $k=2$, there are at most two edges, each of which runs from the vertex $v_{0}$ representing $q_{0}$ to one of the other vertices $v_{1}$ or $v_{2}$. For, since each $f\left(q_{j}\right)$ is a geodesic curve in the negatively-curved surface $Q$, there cannot be two disjoint adjacent minimal $\delta$-thin arcs without some non-simply-connected region of $Q$ separating them.

If $k>2$, we can always find a vertex with at most one edge connecting it to $v_{0}$. For, suppose $v$ is some vertex with more than such one edge, and let $\xi_{1}$ and $\xi_{2}$ be minimal $\delta$-thin arcs represented by two of these edges. 
Consider cutting $Q$ along $\xi_{1} \cup \xi_{2}$. Since $\xi_{1}$ and $\xi_{2}$ have some non-simply connected region of $Q$ lying between them, both components of $Q-\left(\xi_{1} \cup \xi_{2}\right)$ are planar surfaces of Euler characteristic at most zero. Hence each such surface has Euler characteristic whose absolute value is strictly less than $|\chi(Q)|$.

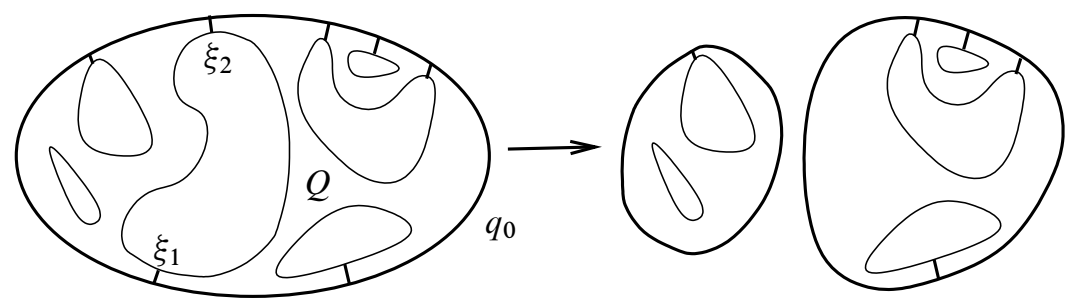

Figure 7: Cutting $Q$ along some $\xi_{1}$ and $\xi_{2}$

Therefore we may argue by induction on $|\chi(Q)|$, choosing one of these new planar surfaces each time. But $|\chi(Q)|$ is finite so this process must terminate. We eventually find a boundary component $q_{j}$ of $Q$ incident with at most one minimal $\delta$-thin arc; equivalently, we find a vertex $v_{j}$ with at most one edge connecting it to $v_{0}$.

Removing this boundary component causes at most one pair of the minimal $\delta$-thin arcs to become adjacent. Since the curvature of $Q$ is negative, these will combine into a single minimal arc.

In the graph, this corresponds to removing the vertex with at most one edge, and amalgamating at most one pair of edges into a single edge. In this way we obtain a graph with one fewer vertex and at most two fewer edges. By induction, $\mathcal{G}_{Q}$ has at most $2(k-1)$ edges.

We have now shown that, for any $0<\delta \leq U$, the surface $R$ has at most $2(k-1) d$ minimal $\delta$-thin arcs. These will provide the possible exceptional cases in the argument which follows in Section 6. Before this, we will determine a couple of constants which will appear in future formulae.

Definition 5.4 For $\Sigma, M$ and $\widetilde{M}$ as described at the beginning of Section 3, recall the set $X_{i}$ of distinguished curves from Definition 3.6.

Consider all geodesic curves $x_{i, j} \in X=\bigcup_{i} X_{i}$. They each have an annular collar in д $\widetilde{M}$ of some strictly positive width $w_{i, j}$. For fixed $i$ these collars are assumed to be disjoint. Define a constant $\epsilon>0$ by $\epsilon=\frac{1}{4} \min _{i, j} w_{i, j}$. We say that $\epsilon$ is the minimum disjoint annular width for $X$. 
It is useful to consider a special case. Choose a boundary component $(\partial \widetilde{M})_{\ell}$ and suppose that each set $X_{i}$ has some boundary curves on $(\partial \widetilde{M})_{\ell}$ in it. By Definitions 3.5 and 3.6 this is equivalent to assuming that, for each $i$, the set $\mathcal{C}\left(\widetilde{\Sigma}_{i}\right) \cap(\partial \widetilde{M})_{\ell}$ is a non-empty proper subset of $(\partial \widetilde{M})_{\ell}$.
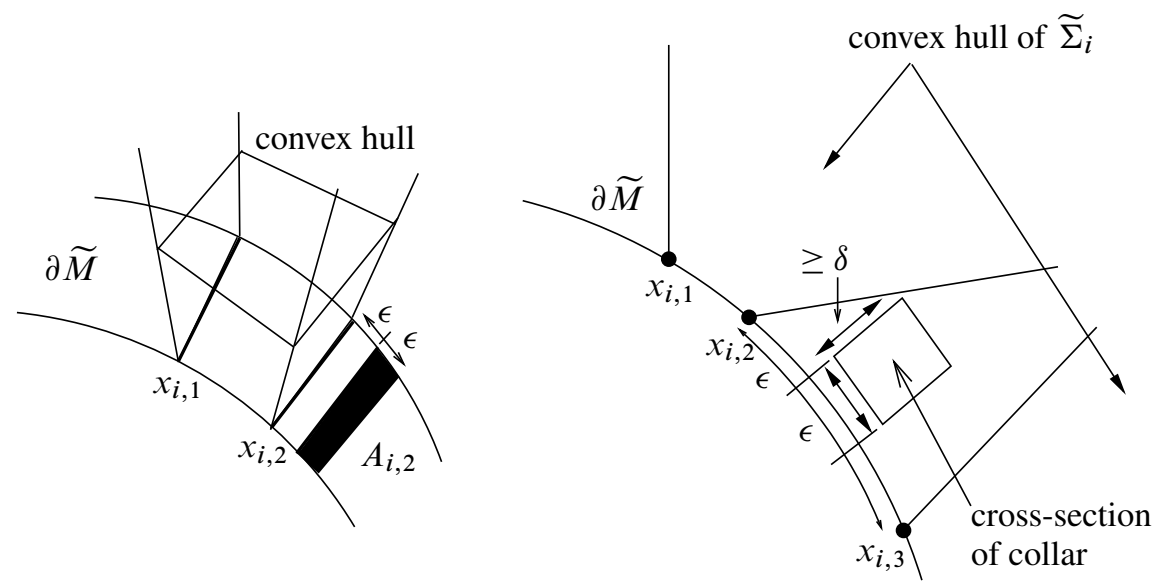

Figure 8: Obtaining a collar disjoint from the convex hull

Choose a lift $\widetilde{\Sigma}_{i}$ of $\Sigma$ and consider its convex hull $\mathcal{C}\left(\widetilde{\Sigma}_{i}\right)$. This intersects $(\partial \widetilde{M})_{\ell}$ in subsurfaces of $\widetilde{M}$ whose boundary curves lie at least a distance $4 \epsilon$ apart. For each boundary curve $x_{i, j}$, consider an annulus $A_{i, j}$ in $(\partial \widetilde{M})_{\ell}$ which lies parallel to $x_{i, j}$ and which is disjoint from the convex hull $\mathcal{C}\left(\widetilde{\Sigma}_{i}\right)$. We assume that the distance between $x_{i, j}$ and the annulus is $\epsilon$, and that the annulus has width $\epsilon$.

Lemma 5.5 There exists a constant $\delta>0$ such that, for all $i, j$, the annulus $A_{i, j}$ has a solid collar $A_{i, j} \times I$ of width at least $\delta$, where this collar is disjoint from the convex hull of $\widetilde{\Sigma}_{i}$.

Proof By taking $\delta \leq U$, we know that each $A_{i, j}$ has a collar of width at least $\delta$ in $\widetilde{M}$. Each annulus $A_{i, j}$ lies outside the appropriate convex hull $\mathcal{C}\left(\widetilde{\Sigma}_{i}\right) \cap(\partial \widetilde{M})_{\ell}$, and there are finitely many such annuli. By compactness for each $i$ and $\ell$, the annuli $A_{i, j}$ lie at least some distance $2 \delta_{i}$ from the appropriate convex hull. Thus they each have a collar of width $\delta=\min _{i} \delta_{i}$ which is disjoint from it.

Take a $\delta$-collar of the boundary of $\widetilde{M}$, and denote it by $N_{\delta}(\partial \widetilde{M})$. By decreasing $\delta>0$ if necessary, the intersection of the convex hull of each $\widetilde{\Sigma}_{i}$ with $N_{\delta}(\partial \widetilde{M})$ is a collar on the intersection of that convex hull with the boundary of $\widetilde{M}$. 
We may do this as explained before Proposition 3.4 by making an extremely small modification (of width $<\delta / 1000$, say) to extend the convex hull to a submanifold with smooth boundary. This condition will ensure that arguments which work for boundary curves also work for any additional curves. We will deal with cut curves separately later.

Definition 5.6 We say that any constant $\delta>0$ satisfying Lemma 5.5 and the above condition on the collar of every convex hull is a minimum collar width for $X$. The value of this constant depends only on $M, \widetilde{M}$ and $\Sigma$.

We have now defined two constants depending on $M, \widetilde{M}$ and $\Sigma$ : the minimum disjoint annular width $\epsilon$ and the minimum collar width $\delta$. These will enable us to prove our main theorem in Section 6. The idea is to show that a planar surface intersects many of these annular collars, and hence picks up at least $\epsilon \sinh \delta$ area each time, leading to a contradiction with the Gauss-Bonnet theorem.

\section{Proof of main theorem: non-cusped case}

We now use the results obtained in Sections 2 to 5 to deduce our main result in the case where the manifold $M$ has no cusps. We consider the general case in Section 7.

Theorem 6.1 Suppose that $M$ is a simple 3-manifold with $m \geq 1$ boundary components $(\partial M)_{1}, \ldots,(\partial M)_{m}$ each of genus $\geq 2$. Consider a collection of handlebodies $\mathcal{H}=\left\{H_{1}, \ldots, H_{m}\right\}$ whose genera match those of $\partial M$. Let $M \cup_{\phi} \mathcal{H}$ denote the closed 3-manifold obtained by gluing each boundary component $(\partial M)_{\ell}$ to $\partial H_{\ell}$ via a homeomorphism $\phi_{\ell}$.

Suppose moreover that $h_{\ell}: \partial H_{\ell} \rightarrow \partial H_{\ell}$ is a pseudo-Anosov homeomorphism with stable lamination of full type. Given homeomorphisms $\phi_{\ell}^{\prime}:(\partial M)_{\ell} \rightarrow \partial H_{\ell}$, there exist integers $\left(N_{\ell}\right)_{\min }$ such that if $\phi_{\ell}=h_{\ell}^{N_{\ell}} \circ \phi_{\ell}^{\prime}$ with $N_{\ell} \geq\left(N_{\ell}\right)_{\min }$ for all $\ell$, the group $\pi_{1}\left(M \cup_{\phi} \mathcal{H}\right)$ contains a surface subgroup.

Recall that $M \neq B^{3}$ is simple if it is compact, irreducible, $\partial$-irreducible, atoroidal and acylindrical, so $\partial M$ is incompressible. A surface subgroup is a subgroup which is isomorphic to the fundamental group of a closed surface of genus at least two. We defined laminations of full type in Definition 2.7.

Proof Any 3-manifold $M \neq B^{3}$ which is irreducible, atoroidal and acylindrical and which has non-empty incompressible boundary is Haken. As a consequence of the 
proof of Thurston's geometrization of Haken manifolds, $M$ can be given a hyperbolic structure with totally geodesic boundary in the sense of Section 1.

By Cooper-Long-Reid [8], there exists a $\pi_{1}$-injective surface $\eta: \Sigma \rightarrow M$. Thus $M$ has a surface subgroup. This surface $\Sigma$ has a finite cover which lifts to a non-peripheral embedded incompressible surface in a finite cover $\pi: \widetilde{M} \rightarrow M$, which we can further assume to be a regular finite cover.

It is therefore enough to show the following theorem. We will make free use of any terminology defined in the preceding sections. We usually abuse notation by writing $\mathcal{H}$ for the union of the handlebodies $H_{1}, \ldots, H_{m}$.

Theorem 6.2 Let $M$ be a hyperbolic 3-manifold with non-empty totally geodesic boundary. Let $\eta: \Sigma \rightarrow M$ be any closed, connected orientable surface with a finite cover which lifts to a non-peripheral embedded incompressible surface in a finite regular cover $\pi: \widetilde{M} \rightarrow M$. Let $M \cup_{\phi} \mathcal{H}$ denote the closed 3-manifold obtained by gluing each boundary component $(\partial M)_{\ell}$ to $\partial H_{\ell}$ via a homeomorphism $\phi_{\ell}$, where each $H_{\ell}$ is a handlebody as in Theorem 6.1.

Suppose moreover that $h_{\ell}: \partial H_{\ell} \rightarrow \partial H_{\ell}$ is a pseudo-Anosov homeomorphism with stable lamination of full type. Given homeomorphisms $\phi_{\ell}^{\prime}:(\partial M)_{\ell} \rightarrow \partial H_{\ell}$, there exist integers $\left(N_{\ell}\right)_{\min }$ such that if $\phi_{\ell}=h_{\ell}^{N_{\ell}} \circ \phi_{\ell}^{\prime}$ with $N_{\ell} \geq\left(N_{\ell}\right)_{\min }$ for all $\ell$, the surface $\Sigma$ stays $\pi_{1}$-injective in the resulting manifold $M \cup_{\phi} \mathcal{H}$.

In particular, $\pi_{1}(\Sigma) \leq \pi_{1}\left(M \cup_{\phi} \mathcal{H}\right)$ is a surface subgroup.

Proof We argue by contradiction. If the surface $\Sigma$ does not remain $\pi_{1}$-injective in $M \cup_{\phi} \mathcal{H}$, there is some essential loop $\mathcal{L}$ in $\eta(\Sigma)$ bounding an immersed disc $f: D \leftrightarrow M \cup_{\phi} \mathcal{H}$. By the hypotheses, we can take each $\left(N_{\ell}\right)_{\min }$ large enough to apply a result of Lackenby [15], and deduce that $\pi_{1}(\mathcal{H})$ injects into $\pi_{1}\left(M \cup_{\phi} \mathcal{H}\right)$. In this case, the intersections of the disc $f(D)$ with $M$ and $\mathcal{H}$ can be assumed to have the following structure.

Lemma 6.3 Suppose $\pi_{1}(\mathcal{H})$ injects into $\pi_{1}\left(M \cup_{\phi} \mathcal{H}\right)$. For any loop $\mathcal{L}$ in $M$ homotopically trivial in $M \cup_{\phi} \mathcal{H}$, take a spanning disc $f: D \rightarrow M \cup_{\phi} \mathcal{H}$ with $\mathcal{L}$ running once round $f(D)$. Assume moreover that $D \cap f^{-1}(\mathcal{H})$ consists of a collection of discs which are disjoint in $D$, and that the number of such discs is minimal. Then $f(D) \cap M$ is a homotopically incompressible and homotopically $\partial$-incompressible surface in $M$.

Note that here homotopically incompressible means that no homotopically non-trivial simple closed curve in $D \cap f^{-1}(M)$ maps to a homotopically trivial curve in $M$; 
homotopically $\partial$-incompressible means that there is no properly embedded essential arc in $D \cap f^{-1}(M)$ which maps to an arc which can be homotoped into $\partial M$ while keeping its endpoints fixed.

Proof We can always find $f: D \rightarrow M \cup_{\phi} \mathcal{H}$ such that $f(D) \cap \mathcal{H}$ is a disjoint union of discs by considering the handlebodies in $\mathcal{H}$ to be small neighbourhoods of their core graphs. Indeed, these graphs can be made to intersect $f(D)$ transversely in isolated points. Thus we can assume that $D \cap f^{-1}(\mathcal{H})$ is a collection of small discs embedded in $D$, and that the number of such discs is minimal.

Suppose $f(D) \cap M$ were homotopically compressible in $M$. Then there would exist a homotopically non-trivial simple closed curve $c$ in $D \cap f^{-1}(M)$ which mapped to a homotopically trivial curve in $M$. We could then modify $f$ by replacing the interior of $c$ by the disc it spans in $M$, reducing $\left|D \cap f^{-1}(\mathcal{H})\right|$. Since this number was assumed minimal, we have a contradiction. Thus we see that $f(D) \cap M$ is homotopically incompressible in $M$.

Similarly, suppose $f(D) \cap M$ were homotopically boundary compressible in $M$. Then there would exist an essential arc $\alpha$ properly embedded in $D \cap f^{-1}(M)$ such that $f(\alpha)$ could be homotoped in $M$ to an arc in $\partial M$ whilst keeping its endpoints fixed.

If such an arc $\alpha$ had both its endpoints on the same boundary component $\partial D_{i}$ of $D \cap f^{-1}(M)$, it would separate $D \cap f^{-1}(M)$ into two planar surfaces as shown in Figure 9. Here $D_{i}$ is a disc component of $D \cap f^{-1}(\mathcal{H})$. After homotoping $f(\alpha)$ into $\partial M$, consider a subdisc $D^{\prime} \subset D$ with image disjoint from $\mathcal{L}$ and with $f\left(\partial D^{\prime}\right) \subset \partial \mathcal{H}$ running along $f(\alpha)$ and a subarc of $f\left(\partial D_{i}\right)$, choosing this subarc so that the disc $D_{i}$ lies inside $D^{\prime}$. This ensures that $\left|D^{\prime} \cap f^{-1}(\mathcal{H})\right| \geq 2$.

Then $f\left(\partial D^{\prime}\right)$ is a curve in $\mathcal{H}$ which is homotopically trivial in $M \cup_{\phi} \mathcal{H}$. By assumption, $\pi_{1}(\mathcal{H})$ injects into $\pi_{1}\left(M \cup_{\phi} \mathcal{H}\right)$, so $f\left(\partial D^{\prime}\right)$ is homotopically trivial in $\mathcal{H}$. This implies that we may modify $f$ so that $f\left(D^{\prime}\right)$ is replaced by a single disc in $\mathcal{H}$. But this too would reduce $\left|D \cap f^{-1}(\mathcal{H})\right|$, a contradiction.

Therefore we must assume that such an $\alpha$ has its endpoints on two different components of $\partial\left(D \cap f^{-1}(M)\right)$, say $\partial D_{1}$ and $\partial D_{2}$ bounding discs $D_{1}$ and $D_{2}$ in $\mathcal{H}$ respectively, as shown in the right-hand diagram of Figure 9.

However, we may then modify $f$ by replacing both discs $D_{1}$ and $D_{2}$ by a single disc $D_{1,2}$ consisting of $D_{1}$ and $D_{2}$ banded together by a strip lying in a small neighbourhood of the image of the arc $\alpha$ on $\partial \mathcal{H}$ pushed into $\mathcal{H}$, as in Figure 10. Note that $\partial D_{1,2}$ is embedded in $D$ although $f\left(\partial D_{1,2}\right)$ is not necessarily embedded in $\mathcal{H}$. This reduces $\left|D \cap f^{-1}(\mathcal{H})\right|$, a final contradiction. 

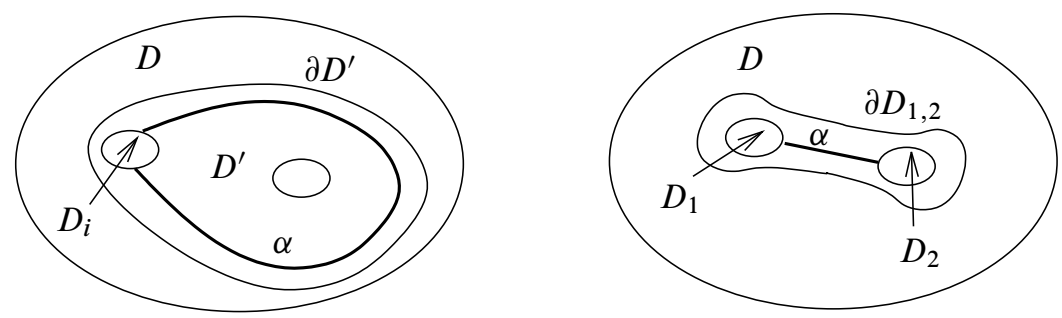

Figure 9: Two possibilities for $\alpha$ : endpoints on the same or different components
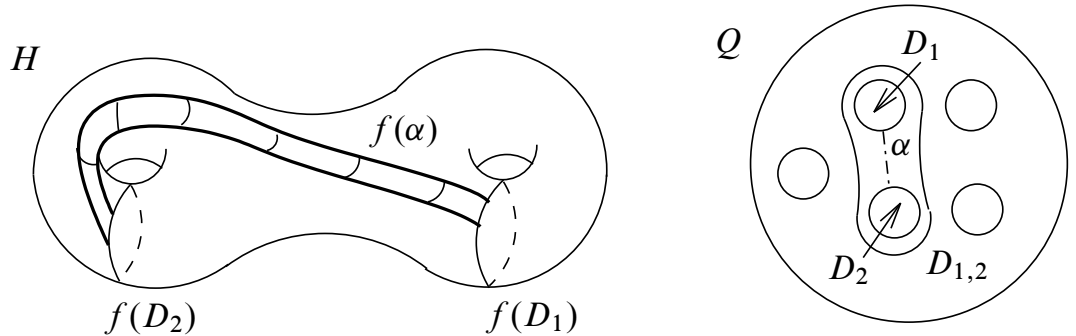

Figure 10: Banding pairs of discs

Thus we have shown that for any curve $\mathcal{L}$ which is homotopically trivial in $M \cup_{\phi} \mathcal{H}$, we can find a spanning disc $D$ such that the planar surface $f(D) \cap M$ is homotopically incompressible and homotopically $\partial$-incompressible.

Proof of Theorem 6.2 By Lemma 6.3, we may consider a least-area immersed planar surface $f: Q \leftrightarrow \rightarrow M$ in the homotopy class of $f(D) \cap M$ ([15], Claim 4). Write the boundary components of $Q$ as $q_{0}, \ldots, q_{k}$. Here $q_{0}$ denotes the boundary component of $Q$ which is mapped to a geodesic curve homotopic to $\mathcal{L}$. The other components $q_{1}, \ldots, q_{k}$ all map to $\partial M$.

We may assume that $k \geq 2$. For, if $k=1$, the essential loop $\mathcal{L}$ is homotopic to a geodesic curve $\mathcal{L}^{*}$ in $\partial M$ bounding a disc in $\mathcal{H}$ under the attaching map. Suppose $\widetilde{\mathcal{L}^{*}} \subset(\partial \widetilde{M})_{\ell}$ is a lift of $\mathcal{L}^{*}$ to the convex hull of $\widetilde{\Sigma}_{i}$, and let $A$ be an annulus realizing the homotopy in $\widetilde{M}$ from $\widetilde{\mathcal{L}}^{*}$ to a lift $\widetilde{\mathcal{L}}$ of $\mathcal{L}$ up to $\widetilde{\Sigma}_{i}$.

The set $X_{i} \cap(\partial \widetilde{M})_{\ell}$ must contain some characteristic curves, else this lift $\widetilde{\mathcal{L}}$ cannot be homotopic to a curve in $(\partial \widetilde{M})_{\ell}$. Proposition 4.1 then shows that $\widetilde{\mathcal{L}}^{*}$ intersects some characteristic curve $x \in X_{i}$ lying in $(\partial \widetilde{M})_{\ell}$. 
By an argument using Johannson's Enclosing Theorem as in Proposition 3.4, some subannulus of $A$ in $\widetilde{M}_{i}^{*} \subset \widetilde{M}$ can be homotoped into the characteristic submanifold $\Psi_{i}$ and hence into $\Psi\left(\partial \widetilde{M}, \widetilde{\Sigma}_{i}\right)$. But this contradicts the assumption that $x$ is a characteristic curve. Thus $k \geq 2$.

Consider a degree $d$ cover $R$ of $Q$ which lifts to $\widetilde{M}$. The images of components of $\partial R$ which cover curves $q_{j}(j \neq 0)$ bound discs under the map $\phi \circ \pi$ and so satisfy the hypotheses of Proposition 4.1. Recall that this ensures that such a curve $\rho \subset(\partial \widetilde{M})_{\ell}$ has many quintersecting subarcs.

Furthermore, note that $R$ has area at most $d$ times that of $Q$, where $d$ is the degree of the cover $\pi: \widetilde{M} \rightarrow M$. Since $Q$ is least-area, the curvature of $Q$ inherited from $M$ is at most -1 (Thm 5.5, $\S$ V.A, [9]). By Gauss-Bonnet,

$$
\operatorname{Area}(R) \leq d \operatorname{Area}(Q) \leq d(2 \pi(k-1))<2 \pi k d .
$$

Recall the minimum disjoint annular width and minimum collar width constants $\epsilon$ and $\delta$, which depend only on $\Sigma, M$ and $\widetilde{M}$. We can use these to deduce a contradiction with the area calculation above by taking

$$
n \geq \frac{2 \pi d}{\epsilon \sinh \delta}+2 d
$$

as the number of quintersecting subarcs; compare Proposition 4.1.

Consider a component $\rho$ of $\partial R$ which projects to some $q_{j}(j \neq 0)$, and the $\delta$-collar $N_{\delta}(\alpha)$ of a quintersecting subarc $\alpha$ of $\rho$. Since $\partial \widetilde{M}$ has a collar of width $U$ in $\widetilde{M}$, and $\delta \leq U$, no $\delta$-collar of another boundary component of $R$ which is a lift of some $q_{j}(j \neq 0)$ may intersect it.

Therefore the only boundary components of $R$ which do intersect $N_{\delta}(\alpha)$ are those which arise as lifts of $q_{0}$. Label such curves $r_{1}, \ldots, r_{t}$. By construction, each $r_{i}$ maps into the convex hull of some lift $\widetilde{\Sigma}_{\sigma(i)}$ of $\Sigma$.

Suppose firstly that the intersection of some set $X_{i}$ of distinguished curves with a boundary component $(\partial \widetilde{M})_{\ell}$ contains no characteristic curves but only an additional curve $x_{\ell}$. Then by our choice of $\delta$ the convex hull $\widetilde{\Sigma}_{i}$ cannot intersect the $\delta$-collar of $(\partial \widetilde{M})_{\ell}$.

In particular, any curves $r_{i_{j}}$ lying in $\mathcal{C}\left(\widetilde{\Sigma}_{i}\right)$ cannot intersect the $\delta$-collar of $\rho$ as it runs along $(\partial \widetilde{M})_{\ell}$ between intersections with $x_{\ell}$. We will see below that this is enough to deduce a contradiction. Henceforth we assume that for all $i$ and $\ell, X_{i} \cap(\partial \widetilde{M})_{\ell}$ contains some characteristic curve. 
Consider an arc of intersection $y$ between a curve $r_{i} \subset \mathcal{C}\left(\widetilde{\Sigma}_{\sigma(i)}\right)$ and $N_{\delta}(\rho)$. It gives rise to a minimal $\delta$-thin $\operatorname{arc} \xi_{y}$ with an endpoint $\zeta$ on $\rho$. Suppose $\alpha$ is a quintersecting subarc of $\rho$ with $y \cap N_{\delta}(\alpha) \neq \varnothing$. If $y$ lies wholly in the $\delta$-collar of the interior of $\alpha$, $\rho$ approaches $y$ most closely at some point in $\alpha$ and $\zeta \in \alpha$.

If instead $\zeta$ lies on a quintersecting subarc $\alpha^{\prime}$ whose interior is disjoint from that of $\alpha$, note that $y$ cannot cross the $\delta$-collar of an annulus $A_{\sigma(i), j}$ parallel to a boundary curve $x_{\sigma(i), j}$ by Lemma 5.5, nor the $\delta$-collar of a cut curve $x_{\sigma(i), j}$ more than twice by Lemma 6.5 below. Assume we have decomposed $\rho$ into $n$ quintersecting subarcs; each of these intersects each characteristic curve five times. Then $y$ can only run through the $\delta$-collar of $\alpha^{\prime}$ and perhaps one quintersecting subarc of $\rho$ adjacent to $\alpha^{\prime}$.

Definition 6.4 We say that a quintersecting subarc $\alpha$ is safe if $\bigcup_{i} r_{i} \cap N_{\delta}(\alpha)$ is either connected or empty.

Each minimal $\delta$-thin arc corresponds to an arc $y$ which intersects the $\delta$-collar of at most two quintersecting subarcs of $\rho$. By Lemma 5.3, $R$ has at most $2(k-1) d$ minimal $\delta$-thin arcs. Therefore, there are at most $2(k-1) d<2 k d$ quintersecting subarcs (in total counting subarcs of all $\rho \subset \partial R$ ) which have two or more arcs $y$ intersecting their $\delta$-collar, and hence are not safe.

Since there are at least $k$ boundary components in $\partial R$ which project to some $q_{j}(j \neq 0)$, and each of these has $n$ quintersecting subarcs, there are at least

$$
n k-2 k d=(n-2 d) k \quad \geq \quad \frac{2 \pi k d}{\epsilon \sinh \delta}
$$

safe quintersecting subarcs. Take a safe quintersecting subarc $\alpha$. At most one component $r_{i} \subset \partial R$ intersects $N_{\delta}(\alpha)$, and it does so in a connected subarc of $r_{i}$. Suppose $r_{i}$ maps to the convex hull of $\widetilde{\Sigma}_{\sigma(i)}$. By Proposition 4.1, $\alpha$ has at least five intersections with every characteristic curve in the set $X_{\sigma(i)}$.

Case I We deal with the simpler of two possibilities first. Suppose that for each boundary component $(\partial \widetilde{M})_{\ell}$ of $\widetilde{M}$, some curves in every $X_{i}$ are boundary curves of $\mathcal{C}\left(\widetilde{\Sigma}_{i}\right) \cap(\partial \widetilde{M})_{\ell}$. That is, not all characteristic curves are cut curves.

Since $\alpha$ is a safe quintersecting subarc, it has at least five (hence at least three) intersections with some boundary curve $x_{\sigma(i), j} \in X_{\sigma(i)}$. Lemma 5.5 then implies that $\alpha$ runs through an annulus $A_{\sigma(i), j}$ of width $\epsilon$ lying outside the convex hull of $\widetilde{\Sigma}_{\sigma(i)}$. The image of $r_{i}$ in $\widetilde{M}$ cannot intersect $N_{\delta}(\alpha)$ while it runs through the collar of this annulus, since the image of $r_{i}$ lies in $\mathcal{C}\left(\widetilde{\Sigma}_{\sigma(i)}\right)$. By assumption, no other boundary 


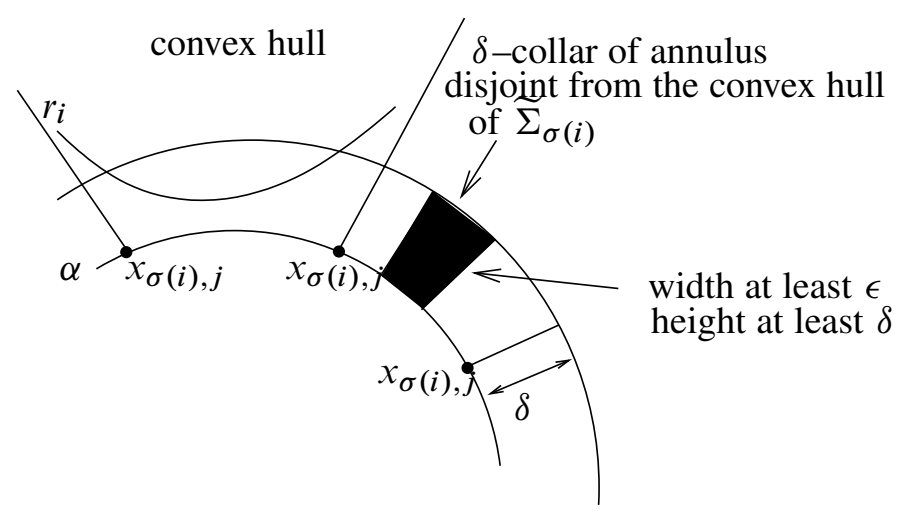

Figure 11: The curve $r_{i}$ cannot run through part of the $\delta$-collar of $\alpha \subset \rho$

component of $R$ can do so either. We deduce that the $\delta$-collar of $\alpha$ contributes area at least $\epsilon \sinh \delta$ to $R$, as illustrated in Figure 11. This concludes the first case.

Case II Now suppose instead that $(\partial \widetilde{M})_{\ell}$ is a boundary component of $\widetilde{M}$ whose intersection with the convex hull $\mathcal{C}\left(\widetilde{\Sigma}_{i}\right)$ of the embedded surface $\widetilde{\Sigma}_{i}$ is all of $(\partial \widetilde{M})_{\ell}$. Since this means that $\mathcal{C}\left(\widetilde{\Sigma}_{i}\right) \cap(\partial \widetilde{M})_{\ell}$ is closed, it has no boundary curves. Thus for such $i, X_{i} \cap(\partial \widetilde{M})_{\ell}$ consists of at least one cut curve.

Recall Definition 3.3: a cut curve $x \in X_{i}$ is a closed geodesic curve on some boundary component $(\partial \widetilde{M})_{\ell}$ such that there exist curves $\sigma^{+}$and $\sigma^{-}$on $\widetilde{\Sigma}_{i}$ which are homotopic to $x$ in $\widetilde{M}_{i}^{*}$ but not to each other in $\widetilde{\Sigma}_{i}$.

These cut curves arose when we insisted that the boundary curves of $\Psi\left(\partial \widetilde{M}, \widetilde{\Sigma}_{i}\right)$ on $(\partial \widetilde{M})_{\ell}$ should be geodesics. Thus for any cut curve $x$ we can find curves $\sigma^{+}$and $\sigma^{-}$in $\widetilde{\Sigma}_{i}$ satisfying the definition and which were boundary components of vertical boundary annuli for $\Psi\left(\partial \widetilde{M}, \widetilde{\Sigma}_{i}\right)$ : see Figure 3 . There may be some Seifert-fibred solid torus components of the characteristic submanifold $\Psi_{i}$ lying between the components of $\Psi\left(\partial \widetilde{M}, \widetilde{\Sigma}_{i}\right)$, but they will make no difference to our argument.

Lemma 6.5 Suppose $r_{i} \subset \partial R$ maps to the convex hull $\mathcal{C}\left(\widetilde{\Sigma}_{\sigma(i)}\right)$, and suppose that $y$ is a connected subarc of $r_{i}$ lying in the $\delta$-collar of $\alpha$. Then this arc $y$ intersects the $\delta$-collar of a cut curve $x_{\sigma(i), j}$ at most twice.

Proof Suppose not, so $y$ intersects the $\delta$-collar of the cut curve $x_{\sigma(i), j}$ three times. Choose one of the endpoints of $y$ as a basepoint for $\widetilde{M}$. Consider the cover $\widetilde{M}_{\infty} \rightarrow \widetilde{M}$ such that $\pi_{1}\left(\widetilde{M}_{\infty}\right)=\pi_{1}\left(\widetilde{\Sigma}_{\sigma(i)}\right)$, and give $\widetilde{M}_{\infty}$ a basepoint which projects to the 
basepoint for $\widetilde{M}$. Note that the manifold $\widetilde{M}_{\infty}$ is homeomorphic to $\widetilde{\Sigma}_{\sigma(i)} \times I$, and moreover that $\widetilde{\Sigma}_{\sigma(i)}$ lifts homeomorphically to $\widetilde{M}_{\infty}$. Write $\widetilde{\Sigma}_{\sigma(i), \infty}$ for such a lift.

Take a $\delta$-collar of the boundary of $\widetilde{M}_{\infty}$, and denote it by $N_{\delta}\left(\partial \widetilde{M}_{\infty}\right)$. By decreasing $\delta>0$ again if necessary (see before Definition 5.6), the intersection of the convex hull of $\widetilde{\Sigma}_{\sigma(i), \infty}$ with this $\delta$-collar is a collar on the intersection of the convex hull with the boundary of $\widetilde{M}_{\infty}$. In this way we obtain

$$
\mathcal{C}\left(\widetilde{\Sigma}_{\sigma(i), \infty}\right) \cap N_{\delta}\left(\partial \widetilde{M}_{\infty}\right)=\left(\mathcal{C}\left(\widetilde{\Sigma}_{\sigma(i), \infty}\right) \cap \partial \widetilde{M}_{\infty}\right) \times I .
$$

Since the cover $\widetilde{M}_{\infty}$ only contains loops which lie in $\pi_{1}\left(\widetilde{\Sigma}_{\sigma(i)}\right)$, the convex hull has been cut along every cut curve in $X_{\sigma(i)}$. Thus every component of the above intersection is the lift of a collar on the appropriate intersection of the original characteristic submanifold $\Psi_{\sigma(i)}$ with $\partial \widetilde{M}$, where we assume that boundary curves on $\partial \widetilde{M}$ remain disjoint rather than being made geodesic.

The loop $r_{i}$ is homotopic to a loop in $\widetilde{\Sigma}_{\sigma(i)}$, and passes through the basepoint of $\widetilde{M}$. When we lift $r_{i}$ to $\widetilde{M}_{\infty}$, it remains a loop based at the chosen lift of the basepoint, lying in the convex hull $\mathcal{C}\left(\widetilde{\Sigma}_{\sigma(i), \infty}\right)$. The subarc $y$ lifts to a subarc in $\widetilde{M}_{\infty}$ lying entirely in one component of the intersection of $\mathcal{C}\left(\widetilde{\Sigma}_{\sigma(i), \infty}\right)$ with $N_{\delta}\left(\partial \widetilde{M}_{\infty}\right)$. Note also that the cut curve $x_{\sigma(i), j}$ is homotopic to a loop in $\widetilde{\Sigma}_{\sigma(i)}$ so that it can be lifted to a loop in $\widetilde{M}_{\infty}$ as well.

Since $y$ crosses the $\delta$-collar of a cut curve $x_{\sigma(i), j}$ three times, we can choose two of these intersections which have coherent orientations. Thus there is a based loop (see Figure 12) lying entirely in one component of $\mathcal{C}\left(\widetilde{\Sigma}_{\sigma(i), \infty}\right) \cap N_{\delta}\left(\partial \widetilde{M}_{\infty}\right)$ which has non-zero geometric intersection with the $\delta$-collar of some lift $\widetilde{x}_{\sigma(i), j}$ of the cut curve. But this component is a lift of a component of

$$
\left(\Psi_{\sigma(i)} \cap \partial \widetilde{M}\right) \times I
$$

and so cannot contain such a based loop crossing the lift of any cut curve. This is a contradiction, proving Lemma 6.5 .

Therefore a curve $r_{i}$ can intersect the $\delta$-collar of at most two intersections of $\alpha$ with $x_{\sigma(i), j}$. Since Proposition 4.1 ensures that $\alpha$ intersects $x_{\sigma(i), j}$ at least five times, there are two consecutive intersections between which $r_{i}$ does not intersect the $\delta$-collar of $\alpha$. By choice of $\epsilon$, these intersections are at least distance $\epsilon$ apart. This ensures that $\alpha$ contributes at least $\epsilon \sinh \delta$ to the area of $R$ between these two intersections, concluding the second case. 


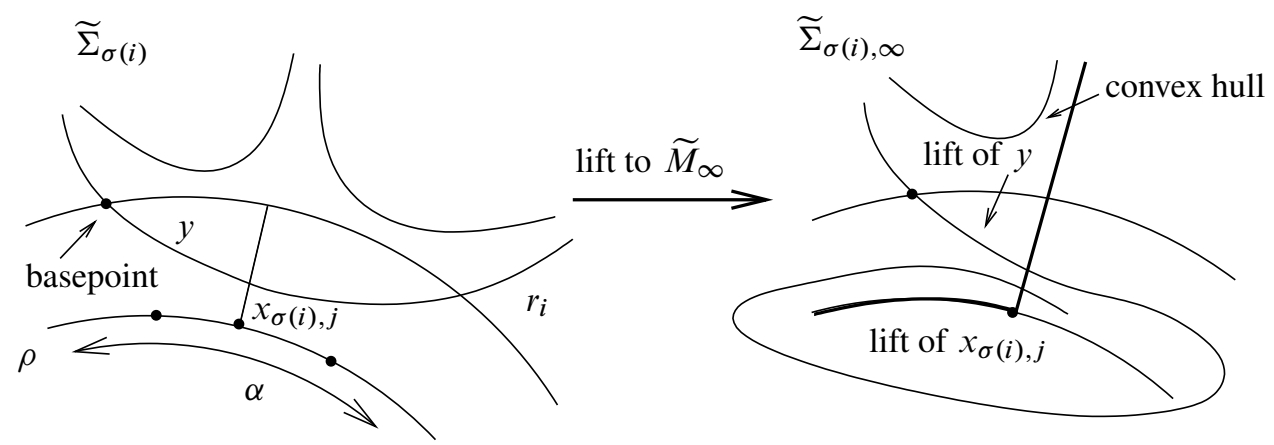

Figure 12: The subarc $y$ of $r_{i}$ and its lift to $\widetilde{M}_{\infty}$

We have shown the existence of at least $2 \pi k d /(\epsilon \sinh \delta)$ subarcs of $\partial R$ each contributing area at least $\epsilon \sinh \delta$ to $R$. This implies that

$$
\operatorname{Area}(R) \geq \frac{2 \pi k d}{\epsilon \sinh \delta} \times \epsilon \sinh \delta \geq 2 \pi k d
$$

which contradicts the Gauss-Bonnet calculation above. Thus no such surface $R$ can exist, and our original assumptions cannot hold. In particular, no essential loop in $\Sigma$ bounds a disc $f: D \leftrightarrow M \cup_{\phi} \mathcal{H}$.

Let us summarize. For a suitable $\pi_{1}$-injective virtually embedded surface $\Sigma$, there exists a constant $n$ as described above depending only on $\Sigma, M$ and $\widetilde{M}$. Choosing suitable pseudo-Anosov maps $h_{\ell}: \partial H_{\ell} \rightarrow \partial H_{\ell}$ and homeomorphisms $\phi_{\ell}^{\prime}:(\partial M)_{\ell} \rightarrow$ $\partial H_{\ell}$, there exist integers $\left(N_{\ell}\right)_{\min }$ depending on $n$ and which have the following property. Whenever $N_{\ell} \geq\left(N_{\ell}\right)_{\min }$ for all $\ell=1, \ldots, m$, the surface $\Sigma$ remains $\pi_{1}$-injective in $M \cup_{\phi} \mathcal{H}$ under the handlebody attachments $\phi_{\ell}=h_{\ell}^{N_{\ell}} \circ \phi_{\ell}^{\prime}:(\partial M)_{\ell} \rightarrow \partial H_{\ell}$. This proves Theorem 6.2.

Proof of Theorem 6.1 By work of Cooper-Long-Reid [8], any 3-manifold $M$ satisfying the hypotheses of Theorem 6.1 contains a surface $\Sigma$ satisfying the assumptions of Theorem 6.2. Indeed, there may be infinitely many such surfaces. They are formed by connecting two copies of an essential surface realizing some boundary slope on $\partial M$ by high-genus tubes wrapping many times round the appropriate components of $\partial M$, and then compressing if necessary.

Theorem 6.2 shows that such surfaces $\Sigma$ remain $\pi_{1}$-injective under appropriate gluing maps $\phi$. In particular, $\pi_{1}\left(M \cup_{\phi} \mathcal{H}\right)$ contains $\pi_{1}(\Sigma)$ as a surface subgroup. This enables us to deduce Theorem 6.1. 


\section{Proof of main theorem: general case}

In Section 6 we proved our main theorem in the case where $M$ does not have cusps. We now show that our arguments also extend to the cusped case.

Theorem 7.1 Suppose $M \neq B^{3}$ is a simple 3-manifold with $m+m^{\prime} \geq 1$ boundary components, $m^{\prime}$ of which are tori. Let $\mathcal{H}=\left\{H_{1}, \ldots, H_{m+m^{\prime}}\right\}$ be a collection of handlebodies and solid tori whose genera match those of $\partial M$. Let $M \cup_{\phi} \mathcal{H}$ denote the closed 3-manifold obtained by gluing each boundary component $(\partial M)_{\ell}$ to $\partial H_{\ell}$ by a homeomorphism $\phi_{\ell}$.

Suppose moreover that $h_{\ell}: \partial H_{\ell} \rightarrow \partial H_{\ell}$ is a homeomorphism which is either a pseudoAnosov homeomorphism whose stable lamination is of full type, or an Anosov homeomorphism, according to whether $H_{\ell}$ has genus at least two or is a solid torus respectively.

Given homeomorphisms $\phi_{\ell}^{\prime}:(\partial M)_{\ell} \rightarrow \partial H_{\ell}$, there exist integers $\left(N_{\ell}\right)_{\min }$ such that if $\phi_{\ell}=h_{\ell}^{N_{\ell}} \circ \phi_{\ell}^{\prime}$ with $N_{\ell} \geq\left(N_{\ell}\right)_{\min }$ for all $\ell$, the group $\pi_{1}\left(M \cup_{\phi} \mathcal{H}\right)$ contains a surface subgroup.

Recall from [6] that an Anosov homeomorphism of a torus is a map whose trace under any geometric $S L(2, \mathbb{Z})$-representation has absolute value strictly greater than two. They fulfil a similar dynamical purpose to pseudo-Anosov maps.

Theorem 7.1 may be deduced from the appropriate analogue of Theorem 6.2 as in the non-cusped case. Since $M$ is Haken, we may give $M$ (minus its toral boundary) a complete hyperbolic structure with totally geodesic boundary.

Theorem 7.2 Let $M$ be a hyperbolic 3-manifold with $m$ totally geodesic boundary components and $m^{\prime}$ cusps, $m+m^{\prime} \geq 1$. Let $\Sigma$ be a connected, orientable, closed, immersed surface in $M$ with a finite cover which lifts to a non-peripheral embedded incompressible surface in a finite regular cover $\pi: \widetilde{M} \rightarrow M$ of degree $d$.

As in Theorem 7.1, let $M \cup_{\phi} \mathcal{H}$ denote the closed 3-manifold obtained by filling each of the boundary components or cusps of $M$ via homeomorphisms $\phi_{\ell}:(\partial M)_{\ell} \rightarrow \partial H_{\ell}$, and consider maps $h_{\ell}$ satisfying the condition given in that theorem.

Given homeomorphisms $\phi_{\ell}^{\prime}:(\partial M)_{\ell} \rightarrow \partial H_{\ell}$, there exist integers $\left(N_{\ell}\right)_{\min }$ such that if $\phi_{\ell}=h_{\ell}^{N_{\ell}} \circ \phi_{\ell}^{\prime}$ with $N_{\ell} \geq\left(N_{\ell}\right)_{\min }$ for all $\ell$, the surface $\Sigma$ remains $\pi_{1}$-injective in the resulting manifold $M \cup_{\phi} \mathcal{H}$. 
Proof of Theorem 7.2 We argue by contradiction as in Theorem 6.2. Apply Lemma 6.3 to a disc $f: D \leftrightarrow M \cup_{\phi} \mathcal{H}$ spanning a loop $\mathcal{L}$ in $\Sigma$, and obtain once more a least-area planar surface $f: Q \leftrightarrow M$ with $k+1$ boundary components and $k^{\prime}$ punctures. We say that the boundary components mapping to totally geodesic boundary components of $\widetilde{M}$ are non-toral curves, and label them $q_{1}, \ldots, q_{k}$. We also label the punctures $p_{1}, \ldots, p_{k^{\prime}}$. Take a cover $\widetilde{f}: R \leftrightarrow \widetilde{M}$ as before.

By the same arguments as for the non-cusped case, we can ensure $k+k^{\prime} \geq 2$. There are then two possible cases: either $\Sigma$ contains accidental parabolics or it does not. Since $\Sigma$ is closed, it contains no genuine parabolics.

Before we examine these cases separately, we need to generalize the proof of one of our results to the cusped case. Recall the proof of Proposition 3.4: at one point we used the assumption that $\pi_{1}(M)$ had no parabolic elements. With this assumption we deduced that $\Psi\left(\partial \widetilde{M}, \widetilde{\Sigma}_{i}\right) \cap \partial \widetilde{M}=\mathcal{C}\left(\widetilde{\Sigma}_{i}\right) \cap \partial \widetilde{M}$.

In fact the same proof works in the cusped case, but we need to justify this. Note that $M$ still cannot be virtually fibred over $\Sigma$ since $\Sigma$ is closed. The only potential problem is the set $P$ of parabolic fixed points which occurs in the identity (Theorem 3.14 of $[18])$

$$
\Lambda\left(\Gamma_{1}\right) \cap \Lambda\left(\Gamma_{2}\right)=\Lambda\left(\Gamma_{1} \cap \Gamma_{2}\right) \cup P
$$

for two geometrically finite subgroups $\Gamma_{1}, \Gamma_{2}$ of a Kleinian group $\Gamma$.

The set $P$ is defined in [18] as the set of points $\zeta$ in the complement $\Omega\left(\Gamma_{1} \cap \Gamma_{2}\right)$ of $\Lambda\left(\Gamma_{1} \cap \Gamma_{2}\right)$ such that $\operatorname{stab}_{\Gamma_{1}}(\zeta)$ and $\operatorname{stab}_{\Gamma_{2}}(\zeta)$ generate a parabolic abelian group of rank 2 and $\operatorname{stab}_{\Gamma_{1}}(\zeta) \cap \operatorname{stab}_{\Gamma_{2}}(\zeta)=\{$ id $\}$.

The set $P$ is only non-empty when the appropriate stabilizers of $\zeta \in P$ are both of rank 1 and intersect trivially. In our case, $\Gamma_{1}$ is the fundamental group of some lift $\widetilde{\Sigma}_{i}$. The group $\Gamma_{2}$ is the fundamental group of some boundary component $(\partial \widetilde{M})_{\ell}$ which is either totally geodesic or cuspidal.

If it is cuspidal, the group $\Gamma_{2}$ contains a rank 2 stabilizer of $\zeta$ if and only if $(\partial \widetilde{M})_{\ell}$ is the appropriate toral boundary component for the cusp corresponding to $\zeta$. If $(\partial \widetilde{M})_{\ell}$ is non-toral or if it corresponds to a different cusp, only the identity element stabilizes the parabolic fixed point $\zeta$. The group $\operatorname{stab}_{\Gamma_{2}}(\zeta)$ can never be of rank 1. In our application, therefore, $P$ is always empty.

We deduce that Proposition 3.4 still holds when $M$ has cusps. Thus, assuming that the characteristic submanifold $\Psi\left(\partial \widetilde{M}, \widetilde{\Sigma}_{i}\right)$ has geodesic boundary on $\partial \widetilde{M}$, the convex hull of $\widetilde{\Sigma}_{i}$ intersects each torus $(\partial \widetilde{M})_{\ell}$ in immersed annuli whose boundary curves are characteristic curves of $\Psi\left(\partial \widetilde{M}, \widetilde{\Sigma}_{i}\right)$ and whose image may be all of $(\partial \widetilde{M})_{\ell}$. 
Now we can return to the main argument. By Gauss-Bonnet applied to $Q$,

$$
\operatorname{Area}(R) \leq d \operatorname{Area}(Q) \leq d\left(2 \pi\left(k+k^{\prime}-1\right)\right)<2 \pi\left(k+k^{\prime}\right) d .
$$

Choose disjoint horoball neighbourhoods of each cusp in $\widetilde{M}$ which are disjoint from the $U$-collars of every non-toral curve. Suppose moreover that the $\delta$-collar of each horoball neighbourhood is disjoint from any convex hull which is disjoint from the toral boundary corresponding to this cusp.

By homotopic $\partial$-incompressibility, each puncture $\tilde{p}$ in $R$ covering a puncture in $Q$ has a half-open annulus neighbourhood $A(\widetilde{p})$ whose image lies in one of these horoball neighbourhoods. The boundary of each horoball neighbourhood is a torus $T_{\ell}$ which inherits a Euclidean path metric from the metric on $\widetilde{M}$.

Case A: no accidental parabolics Suppose $\Sigma$ contains no accidental parabolics: non-trivial loops in $\Sigma$ homotopic to loops in the cusp boundary of $M$. Then, for any lift $\widetilde{\Sigma}_{i}$, the convex hull $\mathcal{C}\left(\widetilde{\Sigma}_{i}\right)$ avoids the cusps of $\widetilde{M}$.

Write $\rho(\tilde{p})$ for the boundary component of $A(\tilde{p})$ on some $T=T_{\ell}$. We say that such curves are toral curves in $R$. By the appropriate and much easier analogue of Proposition 4.1 for these toral attachments, we may ensure that each toral curve $\rho(\tilde{p})$ has uniformly large geometric intersection number with some additional curve $x_{\ell}$. As these intersections occur at least distance $\epsilon$ apart, we may also ensure that all curves $\rho(\tilde{p})$ are longer than any given fixed length.

The area of $A(\tilde{p})$ is at least the length of $\rho(\tilde{p})$ (see e.g. [10], proof of Thm 4.3). By the above argument we can make all toral curves at least $2 d(\pi+\epsilon \sinh \delta)$ long so that each annulus $A(\tilde{p})$ contributes $2 d(\pi+\epsilon \sinh \delta)$ to the area of $R$. There are at least $k^{\prime}$ such annuli.

As in the proof of Theorem 6.2, we can subdivide every non-toral curve into at least $n \geq 2 \pi d /(\epsilon \sinh \delta)+2 d$ quintersecting subarcs. Over all non-toral curves, all but at most $2\left(k+k^{\prime}\right) d$ of these subarcs contribute area $\epsilon \sinh \delta$ to $R$. Thus

$$
\operatorname{Area}(R) \geq\left(\frac{2 \pi k d}{\epsilon \sinh \delta}+2 k d-2\left(k+k^{\prime}\right) d\right) \times \epsilon \sinh \delta+2 k^{\prime} d(\pi+\epsilon \sinh \delta)
$$

and so $\operatorname{Area}(R) \geq 2 \pi\left(k+k^{\prime}\right) d$, which contradicts the Gauss-Bonnet calculation.

In fact, we could have obtained a contradiction by showing that each pair of intersections of $\rho(\tilde{p})$ with $x_{\ell}$ picked up a quantum of area outside $A(\tilde{p})$. We need to use this idea in the general case.

Case B: accidental parabolics Now suppose that the surface $\Sigma$ contains some accidental parabolics. Consider a boundary component $T$ of a horoball neighbourhood of 
Case A

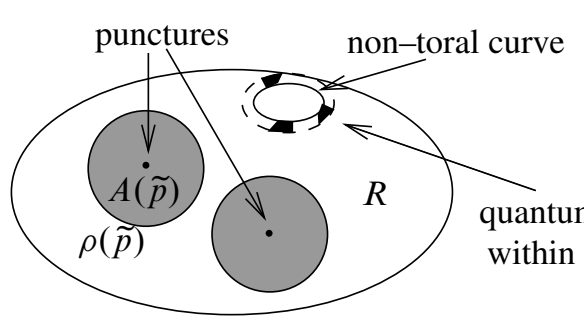

Case B

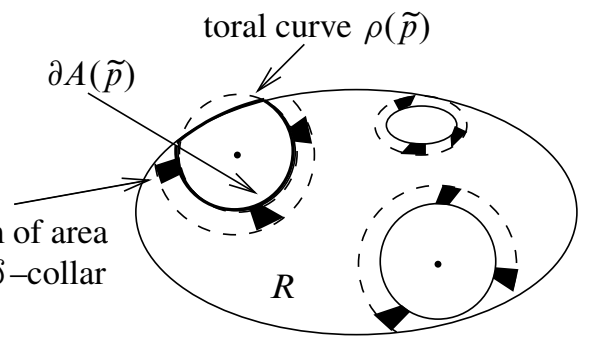

Figure 13: Comparing the area calculations in Case A and Case B

a cusp in $\widetilde{M}$ which has non-empty intersection with some $\mathcal{C}\left(\widetilde{\Sigma}_{i}\right)$, and suppose that $A(\tilde{p})$ lies in the corresponding horoball neighbourhood.

In this case, the boundary curve $\partial A(\tilde{p})$ may not lie entirely in $T$. Define a toral curve $\rho(\widetilde{p})$ instead to be a curve freely homotopic to a core curve of $A(\tilde{p})$ lying on $T$. Also define $N_{\delta}(A(\tilde{p}))$ to be the set of all points in $R$ which lie within $\delta$ of $A(\tilde{p})$. There are two cases.

Case B(I) $\mathcal{C}\left(\widetilde{\Sigma}_{i}\right) \cap T \neq T$ for all $i$ By construction, the only boundary components of $R$ which intersect $N_{\delta}(A(\tilde{p}))$ are those components $r_{i}$ which cover $q_{0}$ and which map to some $\mathcal{C}\left(\widetilde{\Sigma}_{\sigma(i)}\right)$ intersecting $T$.

Since $\mathcal{C}\left(\widetilde{\Sigma}_{\sigma(i)}\right) \cap T$ is never all of $T$, it has some boundary curve $x_{\sigma(i), j}^{\prime}$ on $T$. Again we may adapt Proposition 4.1 to the case of these toral attachments, since they are the composition of a large power of an Anosov map with the map $\phi^{\prime}$. Thus $\rho(\tilde{p})$ can be subdivided into many quintersecting subarcs, each intersecting $x_{\sigma(i), j}^{\prime}$ at least five times. But none of the intersections can occur while $r_{i}$ runs through $A(\widetilde{p})$, so we can replace $\rho(\tilde{p})$ by $\partial A(\tilde{p})$ in this statement.

Taking the powers of the Anosov map in the solid torus attachments to be sufficiently large, we assume that each curve $\partial A(\tilde{p})$ has been subdivided into at least $n \geq 2 \pi d /(\epsilon \sinh \delta)+2 d$ quintersecting subarcs.

By analogy with Definition 5.2, define minimal $\delta$-thin arcs in $R$ to be possibly degenerate minimal arcs of length at most $\delta$ with endpoints on $\partial A(\widetilde{p})$ and some $r_{i}$ respectively. Such an arc may be collapsed to a single point. Adapting Lemma 5.3 to this case, $R$ has $\leq 2\left(k+k^{\prime}\right) d$ minimal $\delta$-thin arcs. Suppose that $\kappa$ of these occur within the $\delta$-collar of a non-toral curve. 
This leaves at most $2\left(k+k^{\prime}\right) d-\kappa$ subarcs of curves $\partial A(\tilde{p})$ which have two or more curves $r_{i}$ intersecting their $\delta$-collar. By the appropriate version of Lemma 5.5, every other subarc is safe and contributes area $\epsilon \sinh \delta$ to $R$.

Subdivide each non-toral curve into at least $n \geq 2 \pi d /(\epsilon \sinh \delta)+2 d$ subarcs. Over all non-toral curves, at most $\kappa$ subarcs fail to contribute area $\epsilon \sinh \delta$. Combining these contributions,

$$
\frac{\operatorname{Area}(R)}{\epsilon \sinh \delta} \geq\left(\frac{2 \pi k d}{\epsilon \sinh \delta}+2 k d-\kappa\right)+\left(\frac{2 \pi k^{\prime} d}{\epsilon \sinh \delta}+2 k^{\prime} d-\left(2\left(k+k^{\prime}\right) d-\kappa\right)\right)
$$

and hence we obtain $\operatorname{Area}(R) \geq 2 \pi\left(k+k^{\prime}\right) d$, contradicting Gauss-Bonnet.

Case B(II) $\mathcal{C}\left(\widetilde{\Sigma}_{i}\right) \cap T=T$ for some $i$ We may combine the arguments above with those explained in Case II of the proof of Theorem 6.2.

This proves Theorem 7.2 and hence Theorem 7.1.

\section{References}

[1] A Abrams, S Schleimer, Distances of Heegaard splittings, Geom. Topol. 9 (2005) 95-119 MR2115669

[2] A Bart, Surface groups in some surgered manifolds, Topology 40 (2001) 197-211 MR1791272

[3] A Basmajian, Tubular neighborhoods of totally geodesic hypersurfaces in hyperbolic manifolds, Invent. Math. 117 (1994) 207-225 MR1273264

[4] F Bonahon, Bouts des variétés hyperboliques de dimension 3, Ann. of Math. (2) 124 (1986) 71-158 MR847953

[5] F Bonahon, The geometry of Teichmüller space via geodesic currents, Invent. Math. 92 (1988) 139-162 MR931208

[6] A J Casson, S A Bleiler, Automorphisms of surfaces after Nielsen and Thurston, London Mathematical Society Student Texts 9, Cambridge University Press, Cambridge (1988) MR964685

[7] D Cooper, D D Long, Some surface subgroups survive surgery, Geom. Topol. 5 (2001) 347-367 MR1825666

[8] D Cooper, D D Long, A W Reid, Essential closed surfaces in bounded 3-manifolds, J. Amer. Math. Soc. 10 (1997) 553-563 MR1431827

[9] S Gallot, D Hulin, J Lafontaine, Riemannian geometry, Universitext, Springer, Berlin (2004) MR2088027 
[10] J Hass, J H Rubinstein, S Wang, Boundary slopes of immersed surfaces in 3manifolds, J. Differential Geom. 52 (1999) 303-325 MR1758298

[11] K Johannson, Homotopy equivalences of 3-manifolds with boundaries, Lecture Notes in Mathematics 761, Springer, Berlin (1979) MR551744

[12] M Kapovich, Hyperbolic manifolds and discrete groups, Progress in Mathematics 183, Birkhäuser, Boston (2001) MR1792613

[13] S P Kerckhoff, The measure of the limit set of the handlebody group, Topology 29 (1990) 27-40 MR1046623

[14] T Kobayashi, Heights of simple loops and pseudo-Anosov homeomorphisms, from: "Braids (Santa Cruz, CA, 1986)", Contemp. Math. 78, Amer. Math. Soc., Providence, RI (1988) 327-338 MR975087

[15] M Lackenby, Attaching handlebodies to 3-manifolds, Geom. Topol. 6 (2002) 889-904 MR1943384

[16] T Li, Immersed essential surfaces in hyperbolic 3-manifolds, Comm. Anal. Geom. 10 (2002) 275-290 MR1900752

[17] H Masur, Measured foliations and handlebodies, Ergodic Theory Dynam. Systems 6 (1986) 99-116 MR837978

[18] K Matsuzaki, M Taniguchi, Hyperbolic manifolds and Kleinian groups, Oxford Mathematical Monographs, The Clarendon Press Oxford University Press, New York (1998) MR1638795

[19] W Menasco, A W Reid, Totally geodesic surfaces in hyperbolic link complements, from: "Topology '90 (Columbus, OH, 1990)", Ohio State Univ. Math. Res. Inst. Publ. 1, de Gruyter, Berlin (1992) 215-226 MR1184413

[20] J W Morgan, H Bass, The Smith conjecture, Pure and Applied Mathematics 112, Academic Press, Orlando, FL (1984) MR758459

[21] W P Thurston, The geometry and topology of 3-manifolds, Princeton University Lecture Notes (1982)

Centre for Mathematical Sciences, University of Cambridge

Wilberforce Road, Cambridge CB3 OWB, UK

V.Easson@dpmms . cam.ac.uk

http://www.dpmms . cam . ac . uk/ vre20

Proposed: Cameron Gordon

Received: 4 March 2004

Seconded: Joan Birman, Colin Rourke

Revised: 19 July 2005 